\title{
ANÁLISIS HISTÓRICO DE LA COMPOSICIÓN QUÍMICA DE FORRAJES TROPICALES EN COLOMBIA ENTRE 1985 Y 2015. I - GRAMÍNEAS FORRAJERAS
}

\author{
HISTORICAL ANALYSIS OF CHEMICAL COMPOSITION \\ OF TROPICAL GRASSES IN COLOMBIA BETWEEN 1985 \\ AND 2015. I - FORAGE GRASSES
}

1 Wilmer Alfonso Cuervo Vivas

2 Luz Elena Santacoloma Varón

${ }^{3}$ Leonor Barreto de Escovar

\author{
${ }_{1}^{1}$ Magister en Ciencias Agrarias Universidad Nacional de Colombia sede Medellín \\ ${ }^{2}$ Doctora en Desarrollo Sostenible de la Universidad Católica de Ávila \\ ${ }^{3}$ Doctora en Desarrollo Sostenible, Sociedad, economía y medio ambiente \\ 1,2,3 Universidad Nacional Abierta y a Distancia \\ 1 1wilmer.cuervo@unad.edu.co \\ 2 luz.santacoloma@unad.edu.co \\ 3 leonor.barreto@unad.edu.co
}

\section{RESUMEN}

Para evaluar dinámicas en composición nutricional de forrajes tropicales utilizados para la producción bovina en Colombia, se adelantó una revisión sistemática en un motor de búsqueda inespecífico de investigaciones publicadas en Colombia entre 1985 y 2015 que incluyeran reportes de fracciones químicas asociadas a calidad forrajera y parámetros productivos animales. Se seleccionaron como componentes: Proteína Cruda, Fibra Detergente Neutra, Fibra Detergente Ácida y Digestibilidad In Vitro de la Materia Seca. También se tuvo presente reportes de ganancia diaria de peso, producción de leche y capacidad de carga. Se realizaron regresiones lineales para cada fracción química y para cada parámetro productivo en el periodo estudiado. De la búsqueda se obtuvieron 105 estudios en 17 bases de datos, de los cuales se seleccionaron especies con más de 5 estudios publicados. No se observaron tendencias lineales significativas en el tiempo para las fracciones químicas analizadas, ni para las variables productivas asociadas a la calidad del forraje. Sin embargo, para Brachiaria sp y Paspalum s.p se advierte una tendencia significativa al aumento en UGG/ha ( $r 2=0,54$ y 0,53 respectivamente). A pesar de la baja significancia de la regresión se observó en las 9 especies, aumento sostenido en PC y DIVMS y reducción en FDA (salvo en Lolium $s p$ y Dichantium $s p$ ). Se identifica 
una ligera tendencia positiva para la capacidad de carga en la mayoría de las especies. Se concluye, que los estudios históricos constituyen una valiosa herramienta para explicar y predecir el comportamiento nutricional de especies forrajeras y su efecto en variables de interés zootécnico.

Palabras clave: Forrajes, Parámetros productivos Revisión, valor nutricional

\section{ABSTRACT}

To evaluate dynamics in nutritional composition of tropical forages used in cattle production in Colombia a systematic research was made, using a regular search engine, for studies published in Colombia between 1985 and 2015, Those studies had to include chemical composition data, such as crude protein, neutral detergent fiber, acid detergent fiber and in vitro dry matter digestibility (CP, NDF, ADF, IVDMD). Likewise studies that reported productive parameters such as daily weight gain (DWG Kg/d), milk yield (MY Lt/d), Animals per hectare (UGG/ha), were included. A lineal regression model was used for chemical components and productive parameters for the period studied. Starting from the global research, 105 studies were included from 17 research database, from which 9 species were selected (species with more than 5 studies published). No lineal trends were observed in the time lapse analyzed, for neither chemicals fractions nor productive parameters related to forage quality. However for Brachiaria $s p$ and Paspalum $s p$ a positive trend was detected for UGG/ha ( $r 2=0.54$ y 0.53 , respectively). Despite the low significance of the coefficients of determination of lineal regression model for the 9 species selected, an overall lineal increase in CP and IVDMD was observed, and also a lineal reduction for ADF (except for Lolium $s p$ and Dichantium $s p$ ). Regarding to productive parameters, a slight positive trend was observed for the UGG/ha in most of the species analyzed. This kind of study becomes in a valuable tool in order to explain and to predict the nutritional behavior of the forage species and its effect on livestock productive parameters.

Keywords: Forage, Productive parameters, Review, Nutritional value

\section{RESUMEN GRÁFICO}
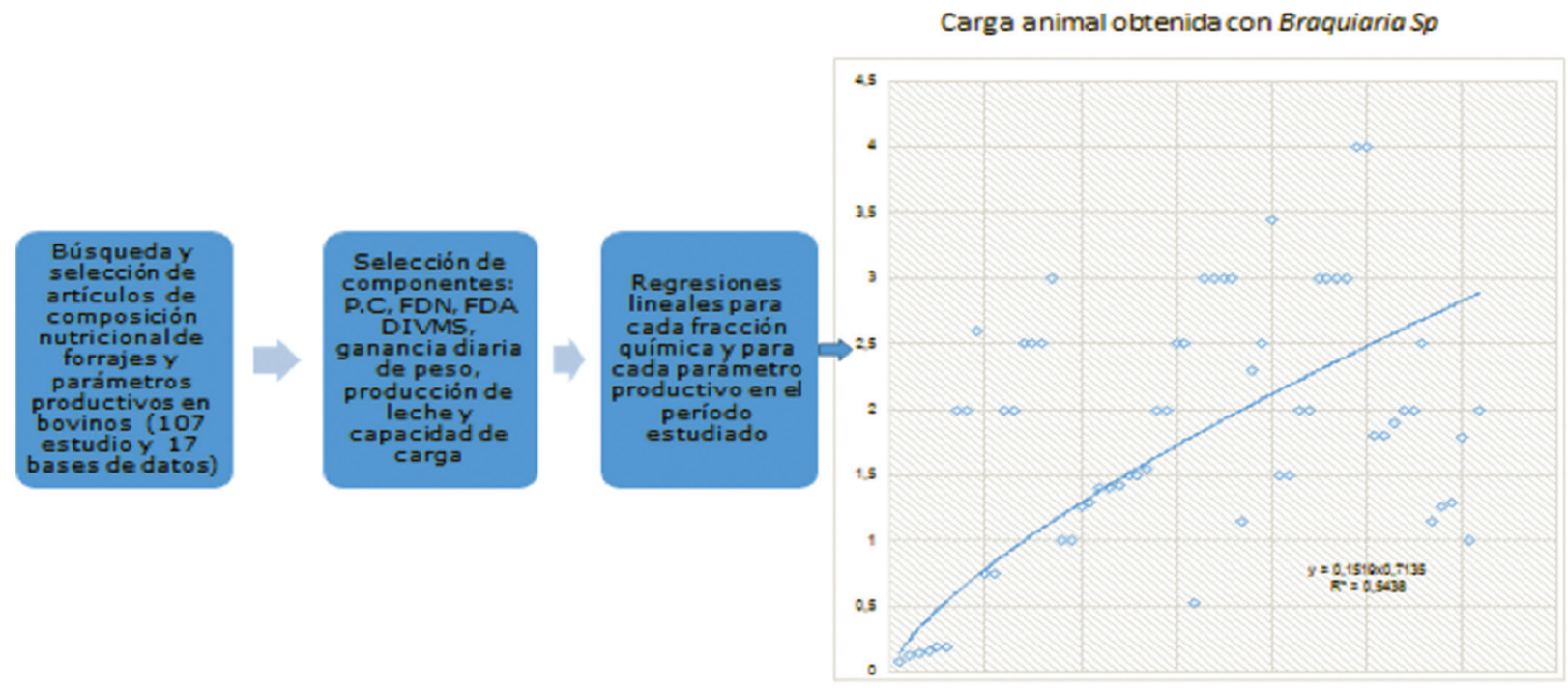


\section{INTRODUCCIÓN}

Los sistemas de producción ganadera en un país tropical como Colombia se basan en el aprovechamiento de una gran oferta forrajera, propia de la diversidad climática, topográfica y edáfica de su territorio. Así, para Heywood (2008), es necesario mantener y conservar esta biodiversidad, toda vez que su pérdida generaría problemas no solo en la alimentación animal sino humana. No obstante, en alimentación animal, el uso de estos recursos, presenta como mayor limitante la fluctuación en producción y calidad, lo que determina en gran parte la respuesta de los animales en producción de leche y carne, como consecuencia de cambios en el consumo y digestibilidad del forraje (Castro, Domínguez, Morales y Huerta 2017).

Lo anterior lo comparten Uvidia, Ramírez, Vargas, Leonard, y Sucoshañay (2015), quienes expresan que la estacionalidad en la oferta de forraje y la variación en su valor nutritivo son factores que limitan la producción animal en las zonas tropicales, y para Elizondo (2017), la calidad de los forrajes está afectada por factores internos como; especie, variedad, edad fisiológica y por factores externos, como; clima, características del suelo, manejo de la fertilización y edad de corte. Para Barbosa et al., (2011), la frecuencia de defoliación es una variable que ha mostrado un efecto significativo en producción y calidad nutricional de un cultivo forrajero y según Denda (2017), la capacidad de recuperación después de la defoliación, y el desarrollo de nueva área foliar, es un factor determinante, en la calidad, y ello depende no solo de factores ambientales como el estado hídrico del suelo, el contenido de nitrógeno, sino también del genotipo de la planta.

En coherencia con lo expuesto, Brizuela, Ferrando y Blanco (2005), consideran que la calidad nutricional depende en gran parte de un adecuado manejo, como es la edad a la cual se cosecha el forraje, lo cual afecta la relación hoja: tallo, que presenta el material, cuando es ofrecido a los animales. Gandara, Borrajo, Fernández y Pereira (2017) expresan, que la edad de corte es el factor que más incide en el valor nutricional de los forrajes, debido a que su incremento, aumenta el contenido de Fibra Detergente Neutra (FDN) y Fibra Detergente Acida (FDA) y disminuye la Digestibilidad de la Materia Seca DIVMS y la Proteína Curda (PC). Santana, Pérez y Figueredo (2010), comparten esta afirmación y consideran que la decisión acerca del momento de cosecha es fundamental en la implementación de estrategias sostenibles para la producción forrajera. Tobía y Villalobos (2004), complementan lo anterior, al exponer que en los tallos se concentra el $60 \%$ de la fibra detergente neutro (FDN) de la planta y en las hojas se concentran el mayor contenido de los carbohidratos no estructurales y minerales.

Se han observado en numerosos trabajos que forrajes pertenecientes a zonas intertropicales de mayor altura presentan mayores digestibilidades, menor contenido de fibra indigerible y en zonas de trópico bajo se presentan generalmente forrajes con menor contenido proteico y mayor de fibra (Balan et al., 2014). Otros componentes climáticos como la humedad en el suelo resultan de particular importancia, a lo cual Del Pozo (2008) señala, que "el crecimiento de los pastos es una función de la humedad disponible en el suelo y que esta, a su vez, varía en dependencia de la cantidad y distribución de las precipitaciones, de la estructura y pendiente de los suelos, de los valores de radiación y temperatura, así como del área cubierta por la vegetación".

La fertilización que se aplica al suelo es otro elemento que se destaca, en el valor nutricional de una especie forrajera, al respecto, 
García, Miranda y Borge (2017) reportan mayores rendimientos de proteína bruta, ceniza y digestibilidad de la materia seca en pasto Brachiaria brizantha, manejado con abono orgánico. Así mismo, el tipo de sistema de producción de forraje, es un factor de importancia en el análisis, al respecto, Treydet et al., (2007) reportan que, en la asociación de gramíneas con leguminosas arbóreas o arbustivas, el comportamiento de las primeras, reporta mayores contenidos de proteína que en monocultivo, como resultado del reciclaje de nutrientes, generado en la fijación del nitrógeno de la leguminosa. Paciullo et al., (2011) aportan elementos al análisis al reportar incrementos en la proteína de la gramínea $B$. decumbens en Sistemas Silvopastoriles, (SSP), con los árboles leguminosos Acacia mangium, A. Angustissima y Mimosa artemisiana, con respecto a la gramínea en monocultivo; lo anterior es atribuido a degradación de la materia orgánica y reciclaje de nutrientes en suelos con sombrío. Este comportamiento no es exclusivo de policultivos de gramíneas con leguminosas, ya que algunos investigadores reportan la toma de nutrientes de gramíneas en asociaciones con plantas no leguminosas como T. diversifolia, las cuales establecen asociaciones con hongos micorrízicos.

Por su parte, la productividad del animal rumiante es un reflejo de la oferta y calidad forrajera que recibe y estas condiciones se reflejan en indicadores como capacidad de carga, consumo de materia seca (CMS), ganancia de peso y producción de leche/día. Respecto a la composición nutricional del forraje, en el consumo de materia seca, esta obedece en gran medida a una serie de variables fisiológicas que reaccionan ante la composición del alimento y a su procesamiento en el organismo animal (Vasquez y Smith 2000)

En coherencia con lo expuesto, el primer factor a considerarse es el llenado físico, que provoca el alto contenido de FDN, su fracción degradable y tasa de fermentación del FDN digestible. Al respecto, Mahecha, Escobar, Suárez y Restrepo (2007), encontraron que, al suministrar especies forrajeras con diferentes contenidos nutricionales, se aprecia mayores consumos, en las que presentan altos contenidos de proteínas, minerales y digestibilidad de la materia seca. Para Rivera et al., (2015), tanto la oferta como la calidad del alimento, brindado a rumiantes, inciden en la producción y calidad de la leche, ya que en glándula mamaria las diferentes fracciones que conforman la leche dependen de los precursores obtenidos en la digestión ruminal e intestinal y la absorción de alimentos. En este contexto, los alimentos y forrajes con un contenido de proteína bruta significativo, estimula el incremento de la producción de leche, sobre todo al inicio de la lactancia, debido a que las vacas están en capacidad de movilizar sus reservas corporales de energía para satisfacer las demandas de nutrientes requeridos para su producción (Milera, 2013).

Así las cosas, tanto la producción de leche, como la ganancia de peso dependen en gran media del consumo, digestibilidad y contenido nutricional de los forrajes, debido a que son aspectos altamente relacionados. De este modo, los principales objetivos de este artículo son; identificar las dinámicas en la calidad nutricional de los forrajes a través del tiempo y explicar las relaciones entre la composición química de los forrajes y el comportamiento de parámetros productivos de la especie bovina, durante el período comprendido entre 1985-2015 en Colombia.

\section{MATERIALES Y MÉTODOS}

Se recopilaron estudios realizados en Colombia durante el período 1985 - 2015, en los cuales se reportaba la composición nutricional de forrajes tropicales en diferentes condiciones 
climatológicas y se estimaba el valor de parámetros productivos relacionados con consumo de materia seca, producción de leche, incrementos de peso y capacidad de carga. Los artículos fueron seleccionados teniendo presentes el tiempo de ventana de observación, el área geográfica del país, las especies forrajeras tanto gramíneas como leguminosas, y forrajeras no leguminosas utilizadas en alimentación animal. En la estrategia de búsqueda se incluyeron 17 bases de datos bibliográficas específicas del área agropecuaria: University of Chicago Journals, AGBios, Embrapa, Academic Search Complete, Agris, Scielo, Science Direct, Agricola, Pubmed, Journal of Animal Science, Animal Production, Journal of Dairy Science, Springer Journal, Scopus, Springer Books, e-books y EBSCO, y documentos y tesis de grado del repositorio de la Universidad Nacional Abierta y a Distancia. Los términos de búsqueda fueron: valor nutricional de forrajes, análisis químico de los forrajes, contenido nutricional de forrajes, forrajes en el trópico y se tuvo presente que las investigaciones tuviesen características similares en diseño de investigación, metodologías de análisis válidas, relevancia de los artículos, y autores reconocidos en el ámbito de la investigación.

Se llevó a cabo la codificación de los estudios, de acuerdo al; piso térmico, temperatura, humedad, especie forrajera, y valores nutricionales de los forrajes relacionados con; producción de materia seca por hectárea (TON MS/HA), materia seca (MS \%), proteína cruda (PC \%), Extracto etéreo (EE \%), Cenizas (CEN $\%)$, materia orgánica (MO \%), fibra cruda (FC $\%)$ fibra detergente neutro (FDN), fibra detergente acido (FDA\%), digestibilidad in vitro de la materia seca (DIVMS \%), celulosa (CEL \%), Hemicelulosa (HCEL \%), lignina (LIG \%), energía bruta (Mcal EB/Kg de MS), energía digestible (Mcal ED/Kg de MS), energía metabolizable (Mcal EM/Kg de MS), energía neta de lactancia (Mcal ENI/Kg de MS), calcio (Ca $\%)$, fosforo (P\%), magnesio (Mg \%), potasio ( $\mathrm{K} \%)$. Del mismo modo, se organizó la información correspondiente a; consumo de MS (CMS Kg/d), peso vivo (PV Kg), eficiencia alimenticia (\%), ganancia diaria de peso (GDP $\mathrm{kg} / \mathrm{d}$ ), producción diaria de leche (Lt leche/d), contenido de grasa en leche (\%), contenido de proteína en leche (\%), solidos totales en leche (\%), carga animal (UGG/ha), intervalo entre partos (IEP días), edad al primer parto (días), tasa de concepción (\%) y peso al destete $(\mathrm{Kg})$. Mediante análisis de regresión lineal, se estableció la relación cuantitativa entre el mejoramiento en la composición química de los forrajes a través del período de tiempo estudiado (1985-2015), así como su relación con los parámetros productivos de sistemas de producción bovina.

\section{RESULTADOS Y DISCUSIÓN}

Luego de considerar factores como el efecto del género y especie de los forrajes, la forma de cosecha o utilización (corte, pastoreo) y el número de estudios incluidos en el proceso de búsqueda (los forrajes con mayor cantidad de registros en cuanto a composición y parámetros productivos), se seleccionaron las siguientes especies:

\section{- Brachiaria spp}

- Megathyrsus máximum

- Andropogon gayanus

- Cynodon spp

- Pennisetum clandestinum

- Lolium multiflorum

- Dichantium aristatum

\section{- Paspalum sp}

Para analizar la relación entre la composición de los forrajes y las variables productivas para los animales incluidos en el análisis, se seleccionó el contenido de PC, FDN, FDA, DIVMS y las variables productivas GDP, Lt/d, UGG/ha. 
En algunos estudios incluidos en la investigación, se reportaban el contenido de Ca, P, MS y LIG, así como los parámetros productivos; porcentaje de grasa, proteína y solidos totales, y se consideró incluirlos en el análisis. Sin embargo, No obstante, por cuestiones de falta de uniformidad de los datos no se tuvieron en cuenta.

El resumen del contenido nutricional promedio de las especies gramíneas de pastoreo, seleccionadas en el estudio durante el período 1985 y 2015 , se presentan en la Tabla 1.

Tabla 1. Contenido Nutricional promedio de principales especies de gramíneas de pastoreo entre 1985 y 2015

\begin{tabular}{|c|c|c|c|c|c|}
\hline Especie & & PC (\%) & FDN (\%) & FDA (\%) & DIVMS (\%) \\
\hline \multirow{4}{*}{ Brachiaria Sp } & Periodo analizado & $\begin{array}{l}1989-2015 \\
(n=47)\end{array}$ & $\begin{array}{l}1989-2015 \\
(n=19)\end{array}$ & $\begin{array}{l}1989-2015 \\
(n=20)\end{array}$ & $\begin{array}{l}1986-2015 \\
(n=49)\end{array}$ \\
\hline & $X \pm D E$ & $7,77 \pm 3,65$ & $69,21 \pm 10,95$ & $41,18 \pm 8,68$ & $58,62 \pm 11,22$ \\
\hline & Tendencia $(r 2)$ & Lineal $(0,08)$ & Lineal $(0,04)$ & Lineal $(0,02)$ & Lineal $(0,18)$ \\
\hline & $\begin{array}{l}\text { Cambio anual } \\
\text { estimado }\end{array}$ & 0,07 & $-0,39$ & $-0,24$ & 0,336 \\
\hline \multirow{4}{*}{$\begin{array}{l}\text { Megathyrsus } \\
\text { máximum }\end{array}$} & Periodo analizado & $\begin{array}{l}1989-2012 \\
(n=22)\end{array}$ & $\begin{array}{l}1989-2015 \\
(n=22)\end{array}$ & $\begin{array}{l}1989-2015 \\
(n=12)\end{array}$ & $\begin{array}{l}1986-2015 \\
(n=19)\end{array}$ \\
\hline & $X \pm D E$ & $8,32 \pm 2,28$ & $68,47 \pm 2,47$ & $40,55 \pm 3,82$ & $51,99 \pm 6,95$ \\
\hline & Tendencia $(r 2)$ & $\begin{array}{l}\text { Polinómica } \\
(0,154)\end{array}$ & Lineal $(0,55)$ & Lineal $(0,571)$ & $\begin{array}{l}\text { Polinómica } \\
(0,287)\end{array}$ \\
\hline & $\begin{array}{l}\text { Cambio anual } \\
\text { estimado }\end{array}$ & $\begin{array}{l}0,069 \times 2- \\
1,2055 x+ \\
55,194\end{array}$ & $-0,355$ & $-0,282$ & $\begin{array}{l}0,0349 x 2- \\
0,5863 x+ \\
53,315\end{array}$ \\
\hline \multirow{4}{*}{$\begin{array}{l}\text { Andropogon } \\
\text { gayanus }\end{array}$} & Periodo analizado & $\begin{array}{l}1987-1995 \\
(n=15)\end{array}$ & - & $\begin{array}{l}1988-1995 \\
(n=8)\end{array}$ & - \\
\hline & $X \pm D E$ & $7,66 \pm 2,84(15)$ & - & $49,53 \pm 4,26$ & - \\
\hline & Tendencia $(r 2)$ & $\begin{array}{l}\text { Polinómica } \\
(0,04)\end{array}$ & - & Lineal $(0,173)$ & - \\
\hline & $\begin{array}{l}\text { Cambio anual } \\
\text { estimado }\end{array}$ & $\begin{array}{l}-0,0353 \times 2 \\
+0,5466 x+ \\
6,2069\end{array}$ & - & 0,725 & - \\
\hline \multirow{4}{*}{ Cynnodon sp } & Periodo analizado & $\begin{array}{l}1991-2013 \\
(n=12)\end{array}$ & $1985-2005(n=7)$ & $1993-2015(n=7)$ & $\begin{array}{l}1993-2015 \\
(n=7)\end{array}$ \\
\hline & $X \pm D E$ & $9,81 \pm 1,91$ & $72,38 \pm 4,28$ & $37,48 \pm 4,49$ & $59,16 \pm 1,77$ \\
\hline & Tendencia (r2) & Lineal $(0,023)$ & Lineal $(0,011)$ & $\begin{array}{l}\text { Polinómica } \\
(0,701)\end{array}$ & Lineal $(0,237)$ \\
\hline & $\begin{array}{l}\text { Cambio anual } \\
\text { estimado }\end{array}$ & 0,079 & $-0,21$ & $\begin{array}{l}-1,0673 \times 2+ \\
7,627 x+26,975\end{array}$ & 0,4611 \\
\hline
\end{tabular}


Continuación tabla 1

\begin{tabular}{|c|c|c|c|c|c|}
\hline Especie & & PC (\%) & FDN (\%) & FDA (\%) & DIVMS (\%) \\
\hline \multirow{4}{*}{$\begin{array}{l}\text { Pennisetum } \\
\text { Clandestinum } \\
\text { (Cenchrus } \\
\text { clandestinus } \\
\text { (Hoechst EX } \\
\text { Chiov) }\end{array}$} & Periodo analizado & $\begin{array}{l}1985-2014 \\
(n=23)\end{array}$ & $\begin{array}{l}1993-2014 \\
(n=20)\end{array}$ & $\begin{array}{l}1993-2014 \\
(n=16)\end{array}$ & $\begin{array}{l}1984-2014 \\
(n=13)\end{array}$ \\
\hline & $X \pm D E$ & $16,7 \pm 4,0$ & $62,31 \pm 6,22$ & $33,83 \pm 8,38$ & $71,42 \pm 11,03$ \\
\hline & Tendencia (r2) & Lineal $(0,079)$ & Lineal $(0,055)$ & Lineal $(0,079)$ & Lineal $(0,053)$ \\
\hline & $\begin{array}{l}\text { Cambio anual } \\
\text { estimado }\end{array}$ & 0,161 & $-0,246$ & $-0,495$ & 0,651 \\
\hline \multirow{4}{*}{$\begin{array}{l}\text { Digitaria } \\
\text { decumbens }\end{array}$} & Periodo analizado & $\begin{array}{l}1985-2010 \\
(n=10)\end{array}$ & $\begin{array}{l}1985-2000 \\
(n=6)\end{array}$ & - & $\begin{array}{l}1984-2014 \\
(n=13)\end{array}$ \\
\hline & $X \pm D E$ & $9,51 \pm 1,71$ & $66,41 \pm 4,13$ & - & $58,27 \pm 4,16$ \\
\hline & Tendencia (r2) & Potencial $(0,123)$ & Lineal $(0,58)$ & - & Lineal $(0,119)$ \\
\hline & $\begin{array}{l}\text { Cambio anual } \\
\text { estimado }\end{array}$ & $8,515 x^{0,1014}$ & 0,525 & - & 1,03 \\
\hline \multirow{4}{*}{ Lolium Sp } & Periodo analizado & $\begin{array}{l}1994-2014 \\
(n=12)\end{array}$ & $\begin{array}{l}1990-2014 \\
(n=12)\end{array}$ & $\begin{array}{l}1984-2014 \\
(n=13)\end{array}$ & - \\
\hline & $X \pm D E$ & $20,51 \pm 4,68$ & $42,17 \pm 10,10$ & $29,5 \pm 4,55$ & - \\
\hline & Tendencia (r2) & $\begin{array}{l}\text { Polinómica } \\
(0,247)\end{array}$ & $\begin{array}{l}\text { Polinómica } \\
(0,536)\end{array}$ & Lineal $(0,114)$ & - \\
\hline & $\begin{array}{l}\text { Cambio anual } \\
\text { estimado }\end{array}$ & $\begin{array}{l}0,2057 x 2- \\
2,8245 x+27,7\end{array}$ & $\begin{array}{l}3,8857 x 2- \\
24,194 x+72\end{array}$ & 0,201 & - \\
\hline \multirow{4}{*}{$\begin{array}{l}\text { Dichantium } \\
\text { aristatum }\end{array}$} & Periodo analizado & $\begin{array}{l}1990-2015 \\
(n=9)\end{array}$ & $\begin{array}{l}1990-2015 \\
(n=8)\end{array}$ & $\begin{array}{l}1990-2015 \\
(n=11)\end{array}$ & $\begin{array}{l}1990-2015 \\
(n=7)\end{array}$ \\
\hline & $X \pm D E$ & $6,44 \pm 0,91$ & $71,32 \pm 2,08$ & $43,87 \pm 8,79$ & $57,85 \pm 21,29$ \\
\hline & Tendencia $(r 2)$ & Lineal $(0,083)$ & Lineal $(0,64)$ & Lineal $(0,48)$ & Lineal $(0,11)$ \\
\hline & $\begin{array}{l}\text { Cambio anual } \\
\text { estimado }\end{array}$ & 0,165 & 1,059 & 0,6455 & 0,68075 \\
\hline \multirow{4}{*}{$\begin{array}{l}\text { Paspalum } \\
\text { notatum }\end{array}$} & Periodo analizado & $\begin{array}{l}1988-2010 \\
(n=6)\end{array}$ & $\begin{array}{l}1988-2010 \\
(n=6)\end{array}$ & - & - \\
\hline & $X \pm D E$ & $8,01 \pm 2,64$ & $74,4 \pm 8,98$ & - & - \\
\hline & Tendencia (r2) & Lineal $(0,369)$ & Lineal $(0,149)$ & - & - \\
\hline & $\begin{array}{l}\text { Cambio anual } \\
\text { estimado }\end{array}$ & 1,001 & $-0,5155$ & - & - \\
\hline
\end{tabular}

Fuente: El estudio 2018

\section{COMPORTAMIENTO HISTÓRICO COMPOSICIÓN QUÍMICA}

\section{- Brachiaria sp.}

Con un total de 47 estudios entre los años 1989 y 2015, reportados en 10 departamentos y 25 municipios ubicados entre los 125 y $1.380 \mathrm{msnm}$, se observó para esta especie una leve tendencia al aumento de PC $(r 2=0,08)$, siendo más evidente un aumento en la DIVMS $(r 2=0,18)$. El comportamiento para FDN y FDA en la ventana de tiempo analizada para esta especie, no arrojó mayores cambios ( $r 2=0,04$ y 0,02 respectivamente). Lo anterior nos indica, un posible mejoramiento en el manejo de la especie a través del tiempo, en cuanto a condiciones ambientales y edáficas.

Como se aprecia en la Figura 1 , se presenta una gran variabilidad en el contenido de proteína durante el periodo analizado sin una 
tendencia significativa (superior al $70 \%$ ) en el comportamiento del contenido de Pc, FDN, FDA o DIVMS.

El comportamiento de las variables productivas reportadas en las investigaciones que incluyeron el uso de Brachiaria $s p$ mostró una tendencia (potencial) al aumento $(r 2=0,54)$ en la capacidad de carga entre 1987 y 1995 (Ver figura 2). No se observaron tendencias significativas para producción de leche ni para la GDP. Este resultado no se asocia con el número de estudios incluidos en este análisis, dado que para GDP se incluyeron 59 artículos y, para UGG/ha, 60. La variación también se explica por qué los datos no se clasificaron de acuerdo a la edad y altura de corte o pastoreo, ante lo cual Beltrán et al., (2005), exponen que esta especie forrajera responde muy bien a la edad de defoliación con mayor producción y calidad de biomasa en sus diferentes órganos.

El alcance del estudio no permite concluir específicamente que el mejoramiento genético realizado sobre las especies del género Brachiaria en los últimos 30 años haya afectado el número de animales que se pueden sostener por hectárea; sin embargo, se hace evidente que, a partir de 1986, hay una tendencia al aumento en este parámetro, tanto que, dentro de la última década, el promedio de UGG/ha aumentó en $19 \%$ con respecto al periodo de 1985-1990. Cabe resaltar el aumento paralelo de la capacidad de carga y el aumento de la DIVMS de este forraje (de $54,1 \%$ en los años 90 a 61,5 \% a final de 2010) en el periodo analizado.

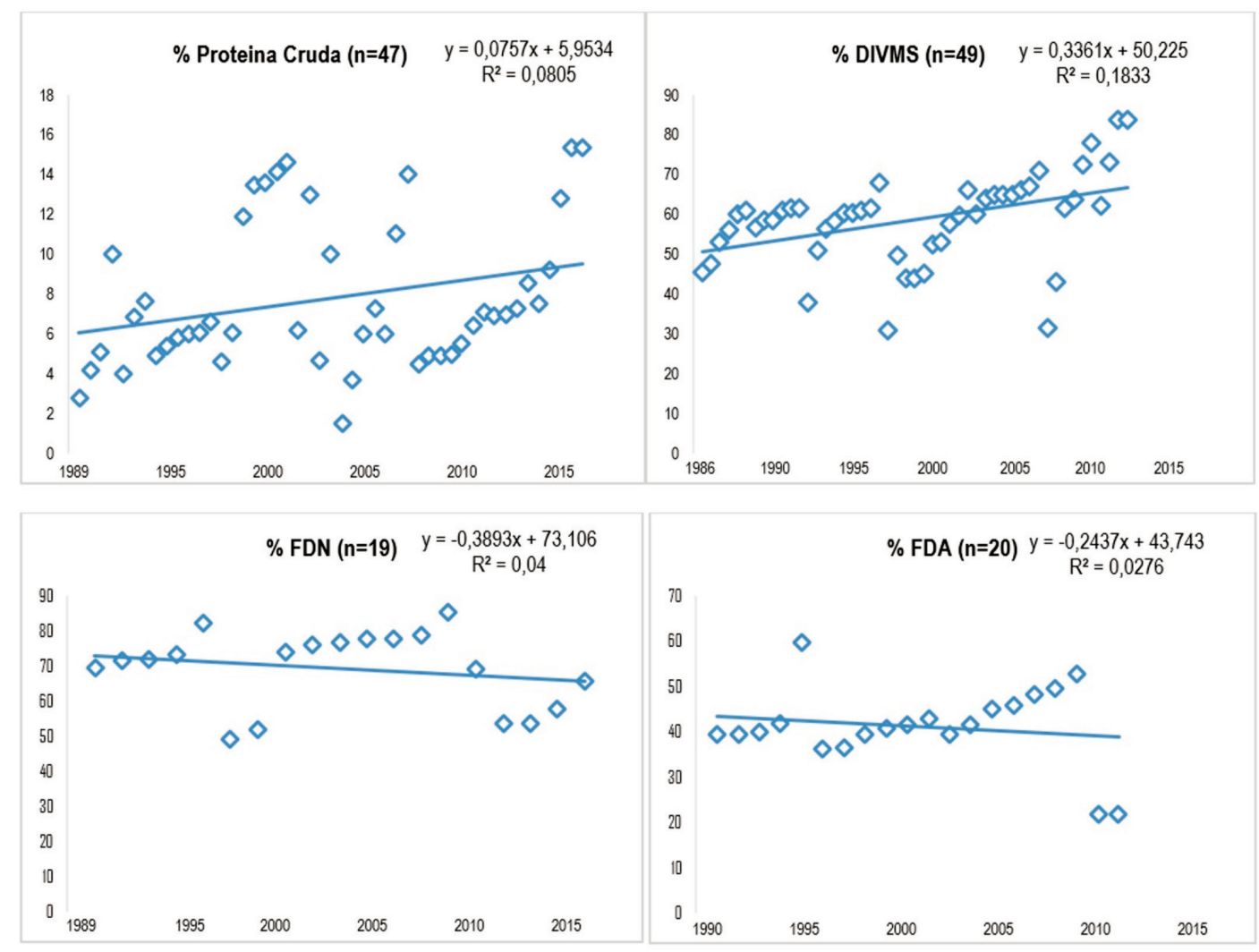

Figura 1. Variación en Composición Química del género Brachiaria Sp entre 1985 y 2015 en Colombia. Fuente: El estudio 2018 
En tal sentido, los factores climáticos per se no controlan de manera lineal el contenido químico de un forraje; sin embargo, elementos como la radiación solar, las precipitaciones y la temperatura ambiente generan una serie de complejas interacciones alterando no solo el contenido sino el rendimiento de los forrajes (Peruchena 2007)

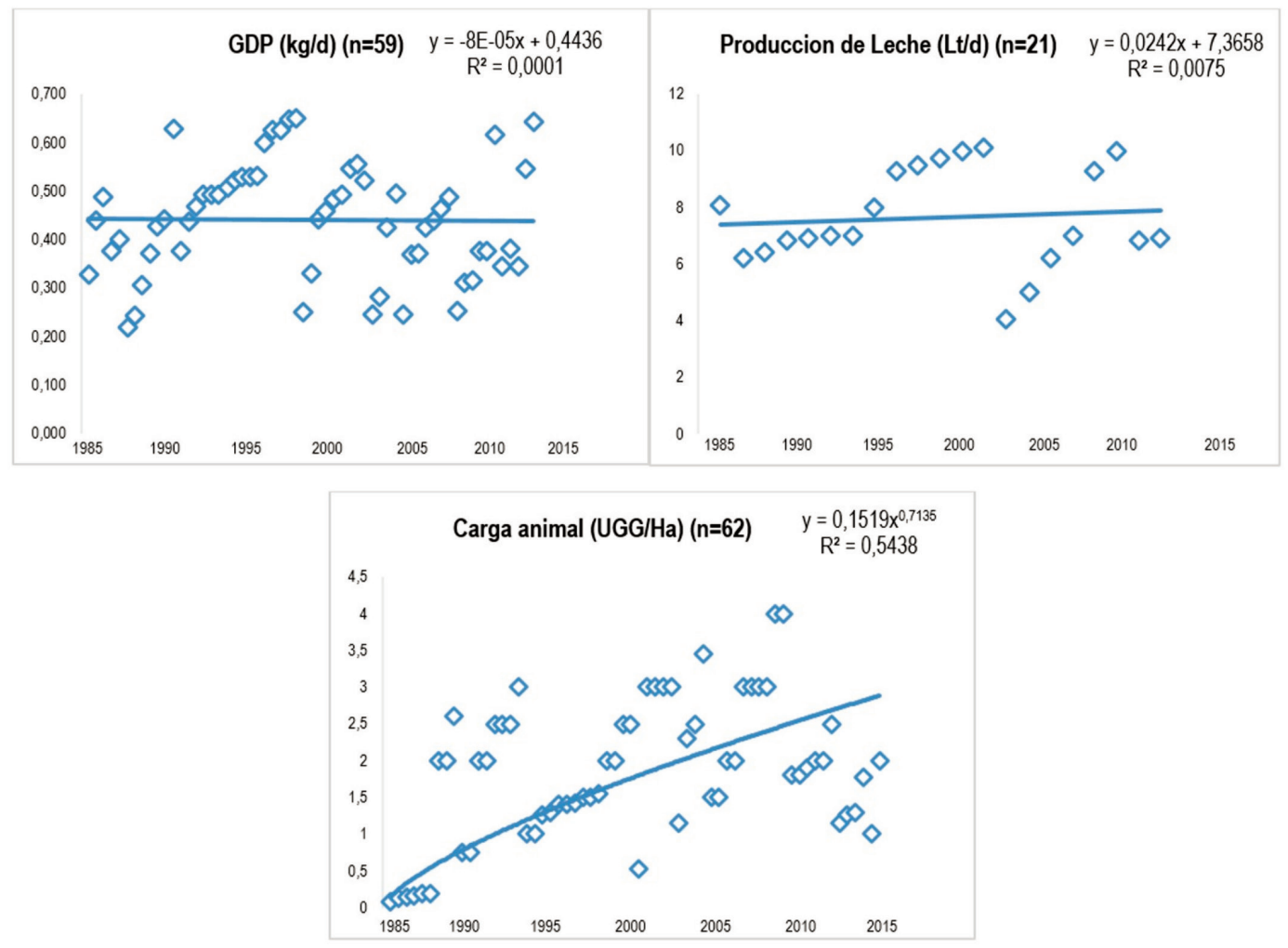

Figura 2. Comportamiento histórico de variables productivas en Bovinos en estudios de Brachiaria sp entre 1985 y 2015 en Colombia.

Fuente: El estudio 2018

- Panicum máximum (Megathyrsus máximum) Con un total de 33 estudios entre los años 1983 y 2013, 10 departamentos y 15 municipios entre los 3 y 2.085 msnm, para esta especie no se observaron tendencias significativas en los contenidos nutricionales, salvo una disminución anual promedio de $1.09 \%$ para FDN $(r 2=0,55)$ y de $0,87 \%$ para FDA ( $r 2=0,51)$ entre 1995 y 2013 . Como se observa en la figura 3 , hay una variabilidad media en el contenido de PC y DIVMS en el periodo analizado, al contrario de la uniformidad encontrada en los datos observados para FDN y FDA. Por su parte, Verdecia et al., (2015), reportan en investigaciones con Megathyrsus maximus, tenores de PC de 14,02 \%, similar a los valores encontrados en los artículos consultados en el presente estudio, para los años comprendidos entre 2012 y 2015 , en tanto que Álvarez et al., (2016), reportan niveles de PC por encima de estos valores, en un estudio en el cual utiliza esta especie en asociación con leguminosas. Lo anterior, indica la alta capacidad de respuesta de la especie, ante la presencia de plantas fijadoras de nitrógeno, como son las leguminosas, las cuales mejoran significativamente la calidad de las gramíneas. 


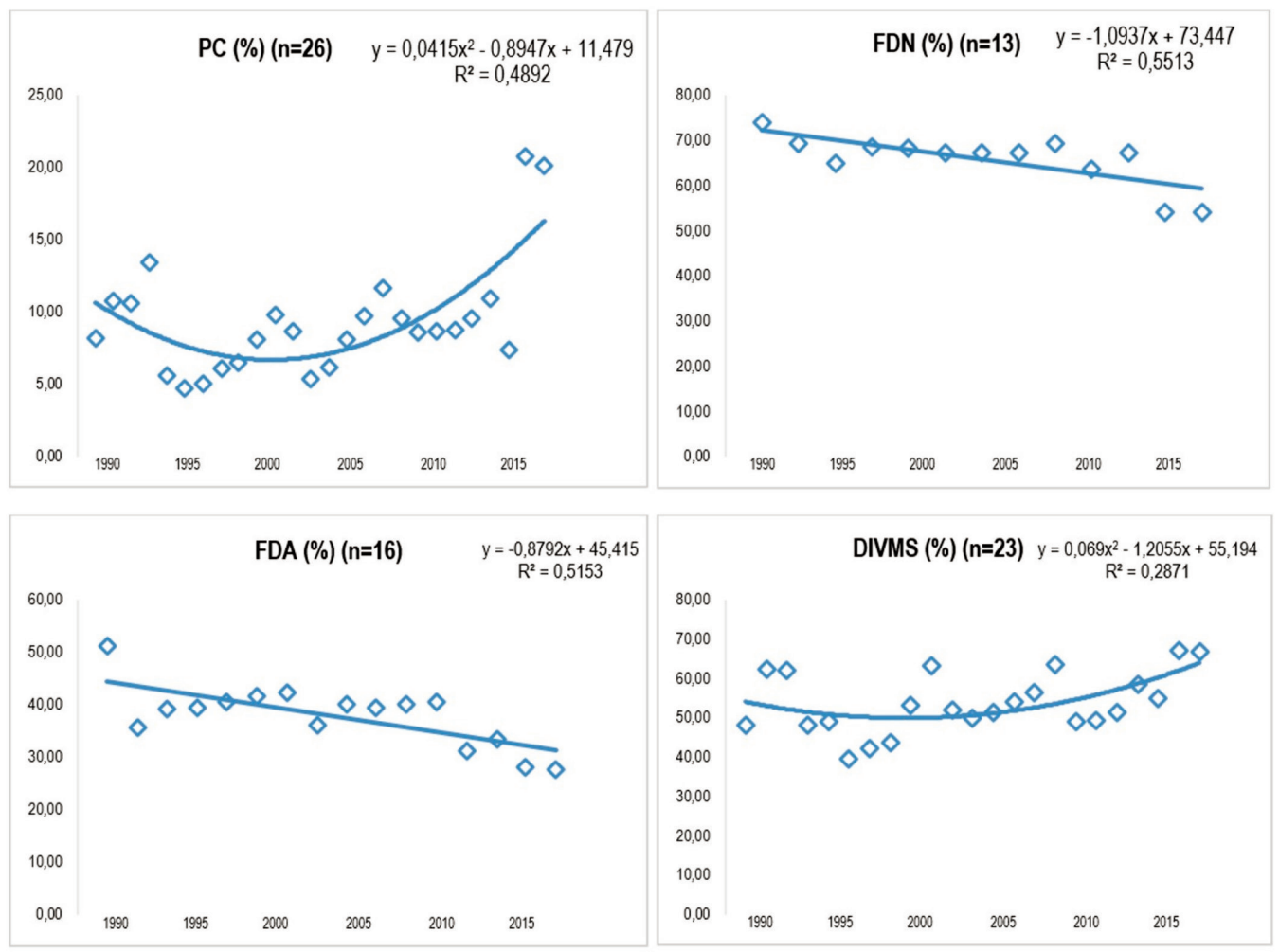

Figura 3. Variación en composición química del genero Megathyrsus máximum entre 1990 y 2015 en Colombia.

Fuente: El estudio 2018

No se observaron tendencias significativas Megathyrsus máximum (lineal para UGG con para el comportamiento de las variables pro- $r 2=0,05$ y potencial para GDP con $r 2=0,13$ ) ductivas reportadas en investigaciones (GDP y entre 1990 y 2015 (Ver figura 4). capacidad de carga) que incluyeron el uso de
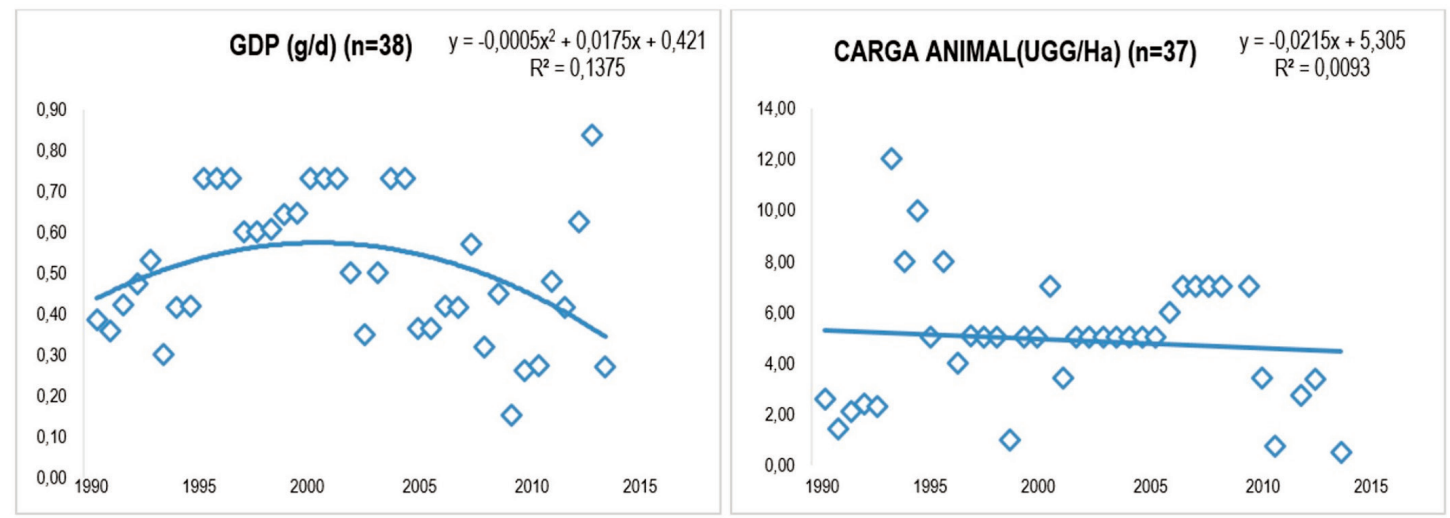

Figura 4. Variación en Ganancia diaria de peso (g/d) y Capacidad de Carga (UGG/ha) en estudios sobre Megathyrsus máximum entre 1990 y 2015 en Colombia.

Fuente: El estudio 2018 
Los niveles históricos de carga animal (UGG/ ha), coinciden con lo reportado por Peñaherrera (2015), con valores de 7,2 t ha-1 y 6,6 t ha-1 con diferentes alturas de corte. Por su parte, al retirar los valores extremos (19, 20 y $30 \mathrm{UGG} / \mathrm{ha}$ ) el comportamiento de la mayor parte de los datos (26 de 37 artículos) muestra que los estudios en los que utilizaron este forraje, es posible sostener 6 o menos animales por hectárea y ello puede estar asociado a su mayor uso como forraje para pastoreo o un menor tiempo de corte para su uso.

\section{- Andropogon gayanus}

Para el Andropogon se tuvo un total de 18 estudios, no obstante, dentro de los mismos el reporte de composición química no fue consistente, de tal manera que los únicos componentes comunes en los estudios fueron el contenido de PC y de DIVMS. Los 18 estudios se realizaron entre los años 1987 y 2013, en 4 departamentos y 8 municipios entre los 365 y $1.071 \mathrm{msnm}$ y se registraron contenidos de PC entre 5 y $11 \%$ y de DIVMS entre 47 y $57 \%$. Estos contenidos de PC resultan bajos para las necesidades de los animales, ante lo cual Ojeda, Pino - Bárbara, Lamela, Santana, y Montejo (2010), señalan que cuando el contenido de proteína bruta del forraje desciende por debajo de $7 \%$, se reduce rápidamente el consumo como consecuencia de deficiencia de nitrógeno en el rumen, lo cual limita la actividad microbiana.

Para esta especie se observó una leve tendencia $(r 2=0,06)$ al aumento de PC $(0,14 \%$ al año), siendo más evidente $(r 2=0,50)$ el aumento en la DIVMS (0,05 \% anual) entre 1989 y 2013 (Ver Tabla 1).

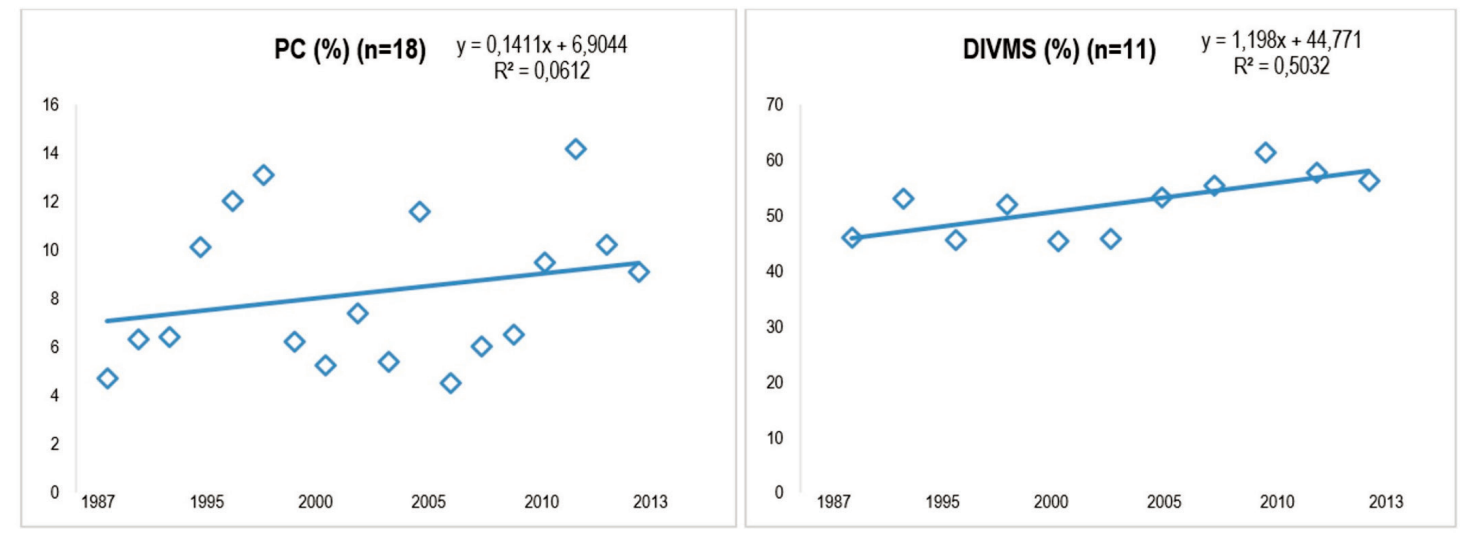

Figura 5. Variación en Fracciones Químicas de Andropogon gayanus en Estudios publicados entre 1987 y 2013 en Colombia.

Fuente: El estudio 2018

Con un total de 12 estudios, el comportamiento de las variables productivas reportadas en investigaciones que incluyeron el uso de Andropogon gayanus no demostraron tendencias significativas para la GDP (lineal r2 $=0,015)$ ni para la capacidad de carga ( $\mathrm{Li}-$ neal $r 2=0,031$ ) en 12 estudios publicados entre 1987 y 2013. 


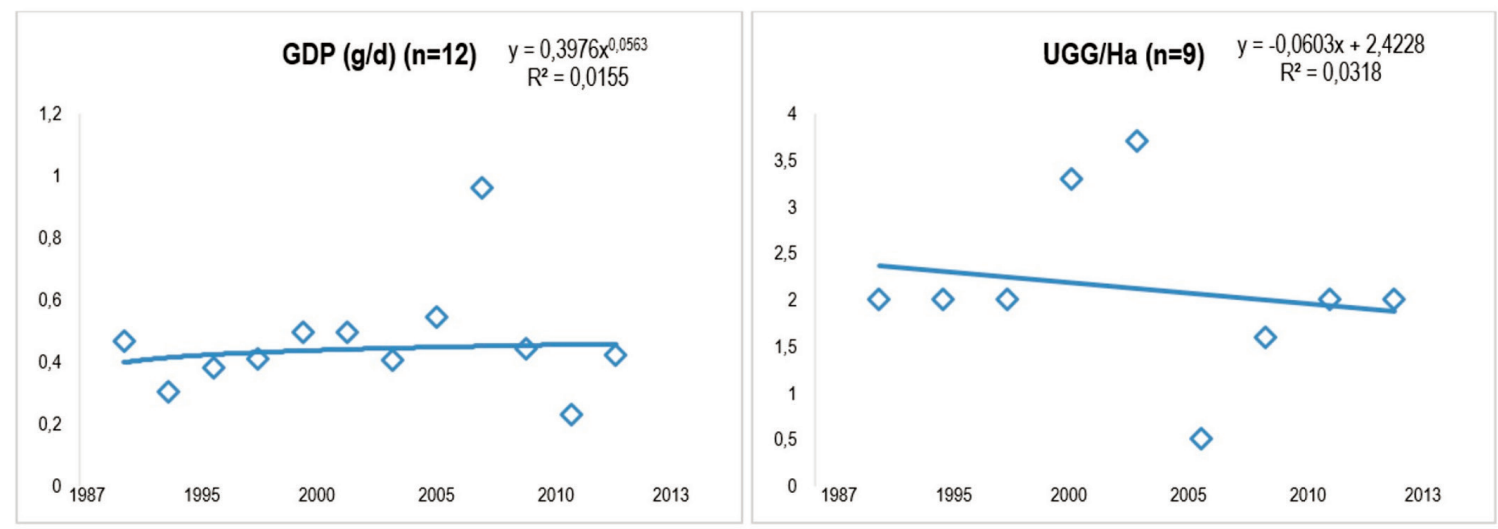

Figura 6. Variación en Ganancia diaria de peso ( $\mathrm{g} / \mathrm{d}$ ) y Capacidad de Carga (UGG/ha) en estudios de Andropogon gayanus entre 1987 y 2013 en Colombia.

Fuente: El estudio 2018

Aunque el alcance del estudio no permite concluir específicamente que un mejoramiento genético realizado sobre Andropogon en los últimos 30 años haya incrementado el número de animales que se pueden sostener por hectárea o la ganancia diaria de peso, sí se advierte una tendencia (aunque leve) en el aumento en la calidad nutricional en cuanto al contenido de PC y del nivel de DIVMS en el periodo analizado. No obstante, Skerman (2003), referencia con esta especie cargas menores de 2,7 UGG, un valor nutritivo relativamente bajo $\mathrm{y}$, en consecuencia, bajos incrementos de peso, aunque destaca palatabilidades superiores al Brachiaria decumbens y Megathyrsus máximum.

\section{- Cynodon sp}

Para el pasto estrella (Cynodon $s p$ ) se revisaron un total de 28 estudios, aunque en los artículos se incluían reportes consistentes para 8 fracciones químicas, únicamente se trabajó con el contenido de PC, FDN, FDA y DIVMS para equiparar con los demás forrajes de este grupo de gramíneas. Los 28 estudios se realizaron entre los años 1985 y 2013, en 8 departamentos y 11 municipios ubicados entre los 150 y 1.760 msnm. En general, se observaron datos medianamente uniformes para PC $(9,81 \pm 1,91 \%)$, FDN $(72,38 \pm 4,28)$, FDA $(37,48 \pm 4,49)$ y DIVMS $(59,16 \pm 1,77)$ en el periodo analizado (1987-2013). Estos datos son consistentes con los expuestos por Juárez, Montero y Núñez (2011) quienes manifiestan que la calidad nutricional del pasto estrella presenta modificaciones rápidamente con la edad y con las variaciones climáticas, lo que reduce su contenido de nitrógeno soluble e incrementa la pared celular. 


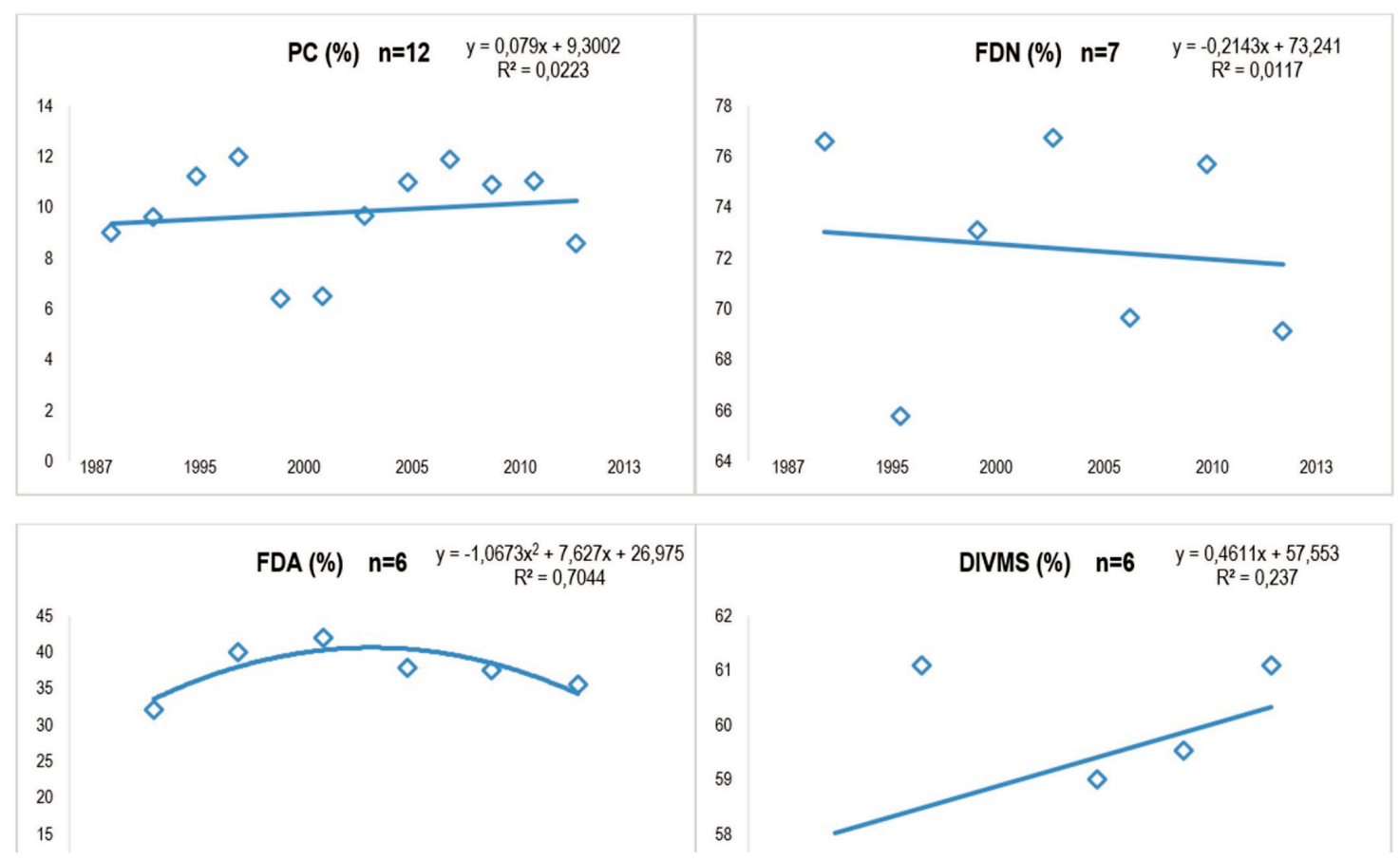

Figura 7. Variación en Fracciones Químicas de Cynodon sp en Estudios publicados entre 1987 y 2013 en Colombia.

Fuente: El estudio 2018

Entre los años 1987 y 2013, para esta gramínea no se observaron tendencias claramente identificables para PC $(r 2=0,02)$ FDN $(r 2=0,01)$ FDA (Polinómica), mientras que para la DIVMS se observó una leve tendencia $(r 2=0.23)$ al aumento $(0,46 \%$ al año) en los últimos 24 años.

Con un total de 22 estudios, el comportamiento de las variables productivas reportadas en investigaciones que incluyeron el uso de $C y$ nodon $s p$ no exhibió tendencias significativas para la GDP (lineal $r 2=0.022$ ) ni para la capacidad de carga (Lineal $r 2=0.04$ ) y una ligera disminución (lineal $r 2=0,32$ ) en el tiempo (0,029 Lt/año) para la producción de leche en estudios publicados entre 1991 y 2015.

Aunque se observa un aumento en la cantidad de animales sostenidos por hectárea en praderas compuestas por esta gramínea (sola o en asocio con leguminosas y otros forrajes), se advierte una ligera disminución en la producción individual de leche, tanto que en los primeros 15 años del análisis (1985 a 2000) se sostenían en promedio 4,25 UGG/ha con una producción individual promedio de 18Lt/d mientras que para la segunda mitad del periodo analizado se observó un reporte de promedio de 6,5 UGG/ha con 10,8 Lt/d. Este bajo comportamiento identificado en los estudios, con base en reportes de León, Martínez, Pedraza y González (2012), se debe, probablemente, a las bajas concentraciones de PC que presenta esta especie en sus diversas etapas de crecimiento y que constituyen un limitante en su calidad nutricional. Esta condición, según Detmann et al., (2004), derivada de bajos niveles de proteína, limita la actividad de los microrganismos ruminales $y$, en consecuencia, afecta la digestibilidad y el consumo de forrajes, causando bajo desempeño en el animal. Al respecto, Torregrosa et al., (2015), afirma que 
la calidad nutricional de una pastura en términos de PC, FDN, FDA y digestibilidad in situ están en función de la edad, parte de la planta cosechada, época del año y estado nutricional del suelo.

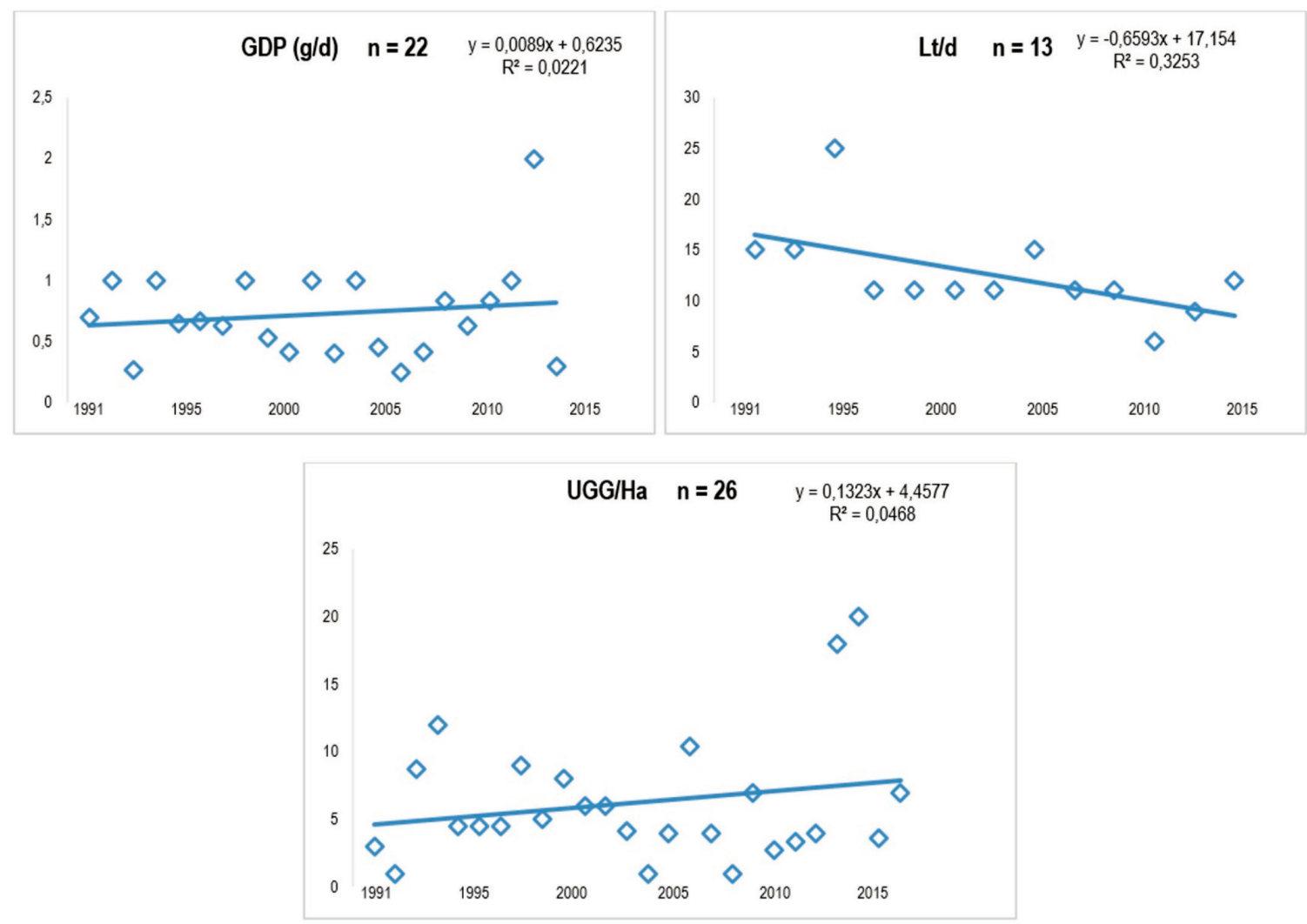

Figura 8. Variación en Ganancia diaria de peso ( $\mathrm{g} / \mathrm{d}$ ), Producción diaria de leche (Lt/d) y Capacidad de Carga (UGG/ha) en estudios de Cynodon Sp entre 1991 y 2015 en Colombia.

Fuente: El estudio 2018

\section{- Pennisetum clandestinum (Cenchrus clandestinus (Hochst. ex Chiov) Morrone)}

Para el Kikuyo se tuvieron en cuenta 23 estudios, publicados en línea (1 uno en físico) entre 1985 y 2014, en los que se encontró que este forraje tuvo, en particular, un alto número de componentes químicos reportados en los estudios incluidos, asociado a su extendido uso en zonas lecheras del altiplano cundiboyacense, nariñense, el norte y oriente antioqueño (cuencas lecheras del país) y, en general, en zonas de ganadería de trópico alto colombiano. Aunque se asume que en la mayor parte de las muestras de kikuyo analizadas en estudios para bovinos contiene un alto nivel de proteína, el análisis del presente estudio reveló una alta variabilidad $(16,7 \pm 4,0 \%)$, del mismo modo, que para el contenido de FDA (33,8 $\pm 8,3 \%)$. Las altas variaciones identificada en la PC ya han sido reportada en varios estudios, como los de Soto, Valencia, Galvis y Correa (2005), quienes expresan que el $P$ clandestinum presenta alta acumulación de $\mathrm{N}$ en los órganos vegetativos en las primeras etapas de crecimiento de los cultivos y disminuye en la etapa de senescencia. 
Los valores relativamente altos de PC encontrados en el estudio, al analizar esta especie, se atribuyen a los intensos planes de fertilización nitrogenada a los cuales son sometidos estas praderas en las cuencas lecheras tanto en el altiplano cundiboyacense, como en el oriente antioqueño (Carulla, Cárdenas, Sánchez y Riveros 2004 y Correa 2011). Por su parte, los niveles promedio de FDN (62,31 $\pm 6,22 \%)$ y DIVMS $(71,42 \pm 11,03 \%)$ reportados para este forraje en el periodo analizado fueron relativamente constantes (Figura 9).

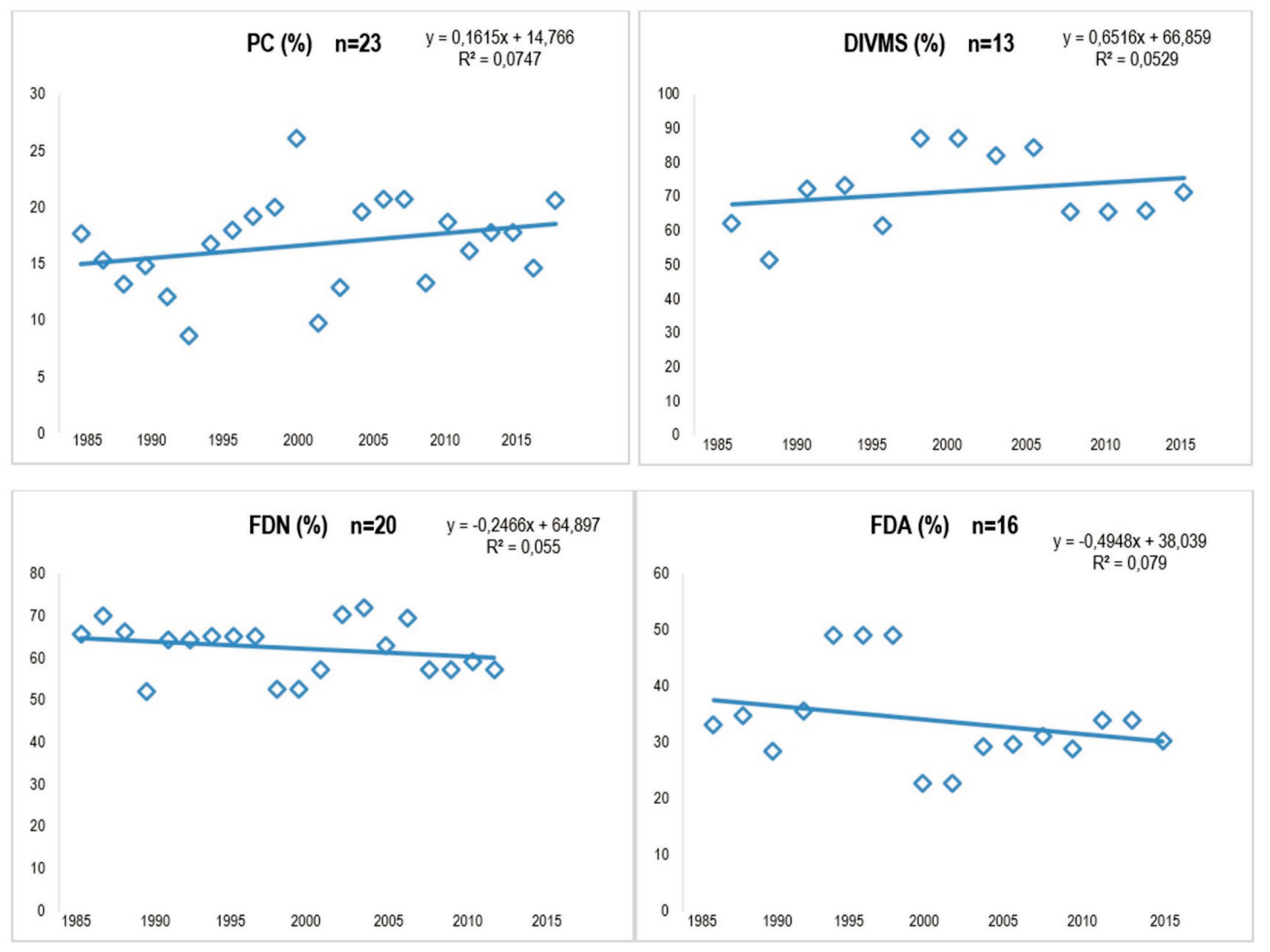

Figura 9. Variación en Fracciones Químicas de Cenchrus clandestinus (Hochst. ex Chiov) en Estudios publicados entre 1987 y 2013 en Colombia.

Fuente: El estudio 2018

En los reportes analizados, no se observaron tendencias significativas a lo largo del periodo 1985 - 2015 para PC $(r 2=0.07)$ DIVMS $(r 2=0.05)$, FDN $(r 2=0.05)$, FDA $(r 2=0.07) . A$ pesar de la no significancia en el análisis de tendencias, si se observó un ligero aumento en el reporte del contenido de PC y DIVMS, así como una leve reducción en el contenido de FDN y FDA. En cuanto al comportamiento de las variables productivas, no se mostraron tendencias estadísticamente significativas en cuanto a su comportamiento en el tiempo analizado. 


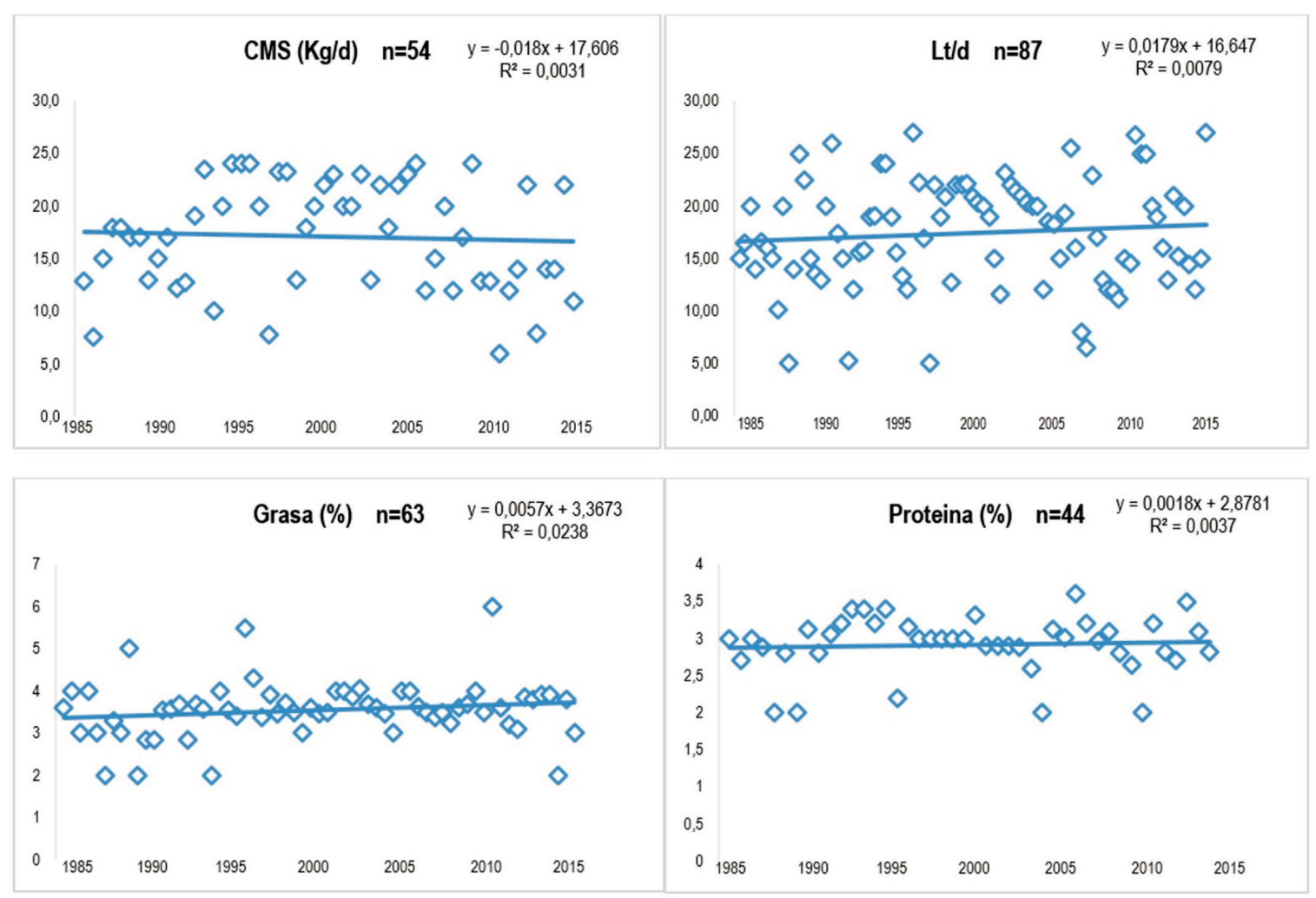

Figura 10. Variación en Consumo de materia Seca (Kg MS/d), Producción de leche (L/d), grasa en leche (\%) y proteína en leche (\%) 1985 y 2015 de Cenchrus clandestinus (Hochst. ex Chiov) en Colombia.

Fuente: El estudio 2018

Es importante mencionar que, para este forraje en particular, fue necesario cambiar las variables productivas analizadas, dado que su utilización en Colombia ha estado ligada a la producción lechera especializada, como el altiplano cundiboyacense, norte y oriente antioqueño y altiplano nariñense. En estos sistemas de producción, variables como el consumo de MS al día, producción y composición de leche son más analizadas que cualquier otro parámetro en estudios publicados. Al respecto Correa (2011), afirma que las características nutricionales más limitantes del pasto kikuyo para la producción de leche es la relación energía: proteína, debido a que si bien el forraje, presenta alto contenido de PC, muestra bajo contenido de Carbohidratos No Estructurales (CNE) y altos componente FDN.

\section{- Lolium multiflorum}

El Raygrass es uno de los forrajes más utilizados en ganaderías especializadas (leche y carne) en trópico alto colombiano, en este sentido, tanto las fracciones químicas más comunes como las variables productivas más analizadas varían con otras gramíneas de pastoreo incluidas en el estudio. No se observaron tendencias lineales en el tiempo para PC, FDN y un comportamiento lineal no significativo ( $r 2=0,014)$ para FDA, con un contenido medio de $20,5 \%, 42,17 \%$ y $29,5 \%$ respectivamente para el periodo analizado. 


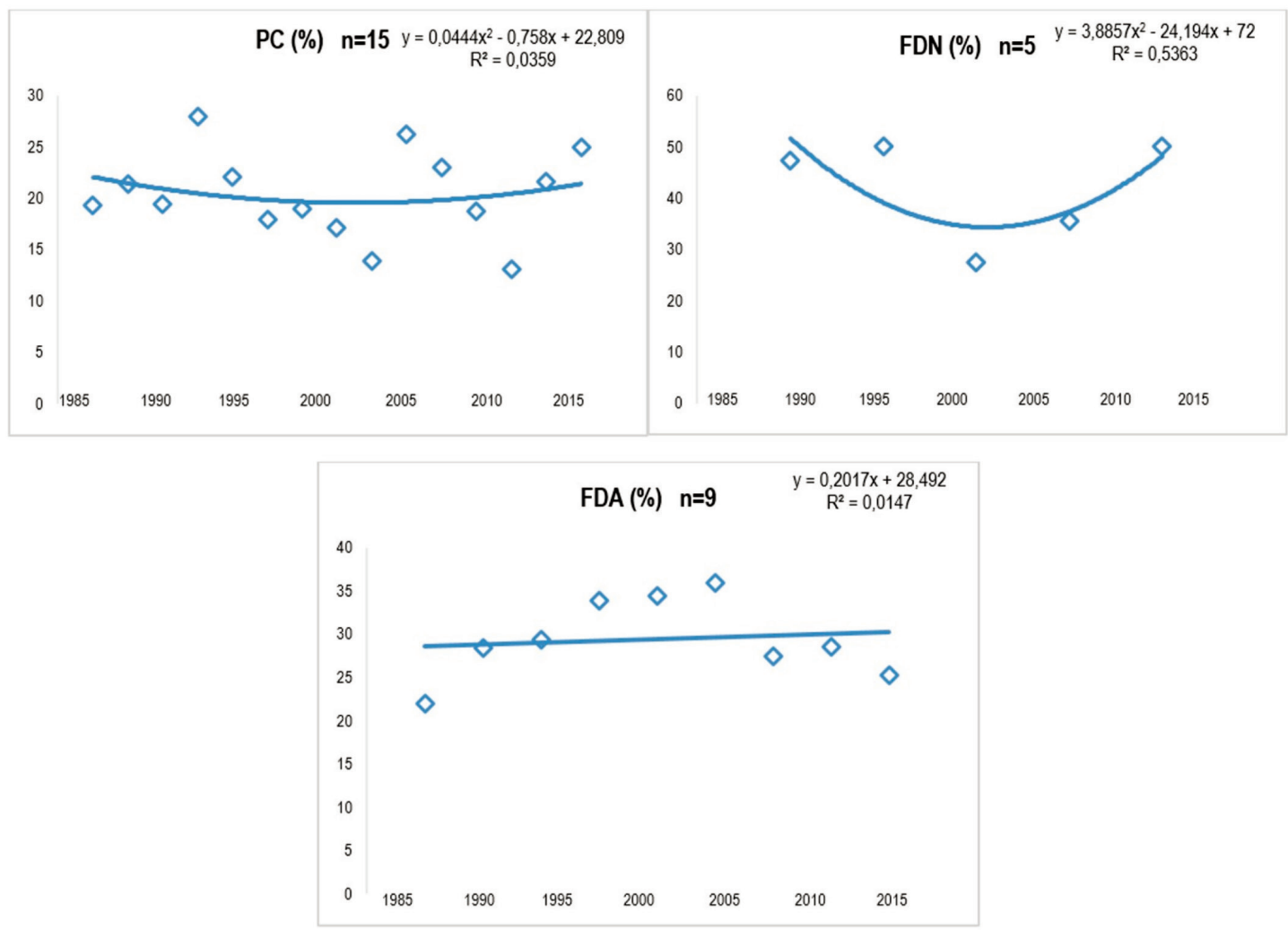

Figura 11. Variación en Fracciones Químicas de Lolium Sp en Estudios publicados entre 1985 y 2015 en Colombia. Fuente: el estudio 2018

El promedio de PC que se obtuvo en el tiempo de observación, para el Lolium multiflorum supera los resultados obtenidos en estudios adelantados por Anrique (2014), donde el contenido de este componente, para una pradera fertilizada fue cercano a $16,7 \%$. No obstante, coincide con los datos reportados por Posada, Cerón, y Arenas, (2013), con valores de 18 a $20 \%$ en pasturas Raigrases de buena calidad, en periodos de lluvia Lo anterior explica la inclusión de esta especie forrajera en rumiantes de alta producción en ganaderías lecheras de trópico alto.

Con respecto al FDN, los resultados promedio encontrados en el estudio son más bajos que los reportados por Villalobos y Sánchez
(2010), en Raygrass tetraploide quien relaciona un $70,2 \%$ de este componente, en condiciones de verano y 49,76 \% en época de sequía, datos que señalan la vulnerabilidad de esta especie frente a cambios climáticos. Al respecto, Veloza (2008), expone que el Raygrass en época seca, desarrolla un gran contenido de FDN por una maduración rápida que genera una prefloración "espiga" acelerada por la falta de pluviosidad y estrés calórico. Los datos de CMS en estudios que incluían el uso de Raygrass mostraron una ligera tendencia positiva $(r 2=0,18)$, no así, la GDP que mostro datos inferiores en los estudios publicados entre 2005 y 2012, sin llegar a ser un comportamiento estadísticamente significativo. 


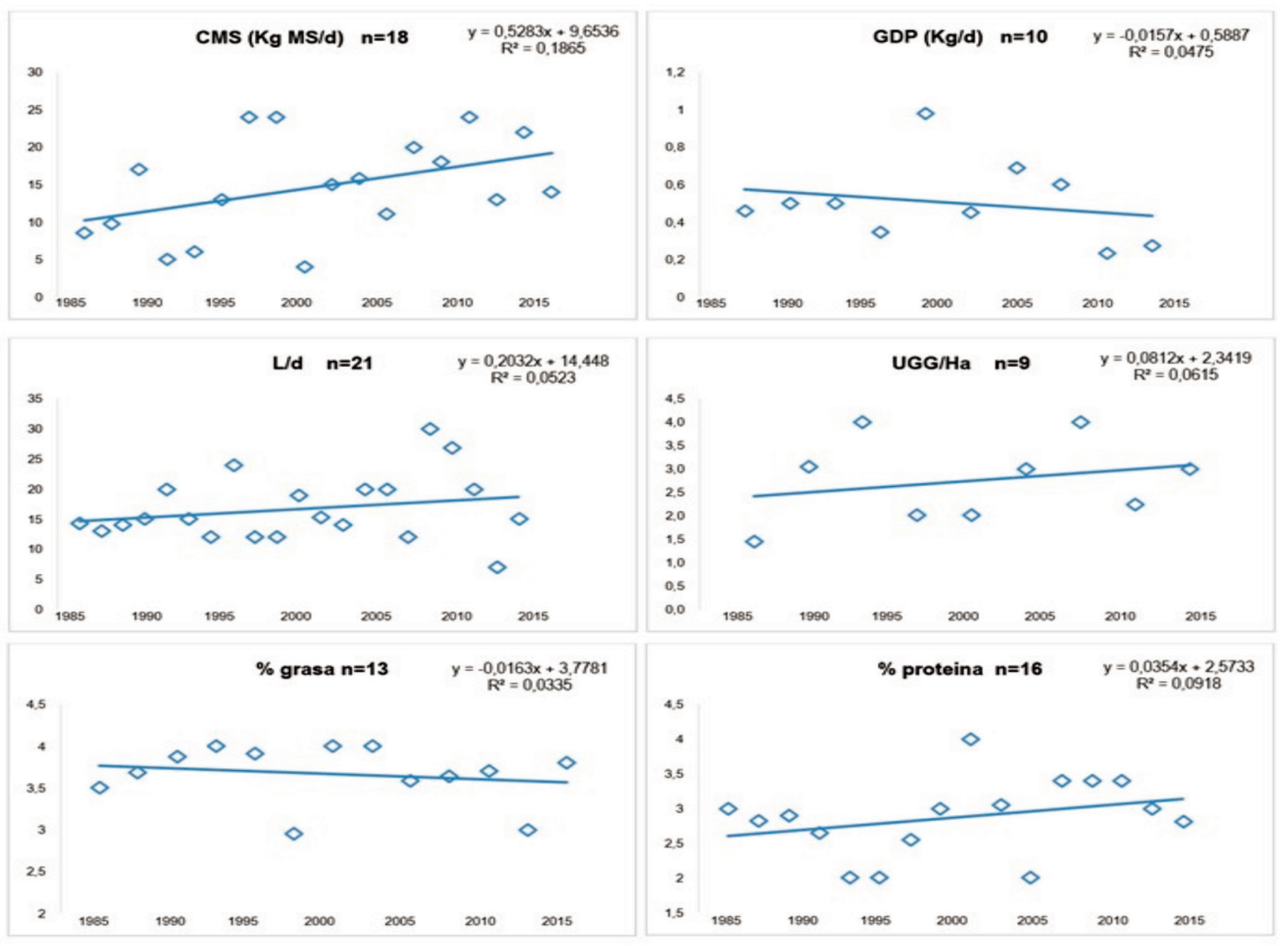

Figura 12. Reporte del nivel de Consumo de materia Seca (Kg MS/d), Ganancia diaria de peso (Producción de leche (L/d), capacidad de carga (UGG/d) grasa y proteína en leche (\%) en investigaciones de Lolium Sp entre 1985 y 2015 en Colombia.

Fuente: El estudio 2018

Ni la capacidad de carga, ni los principales componentes de la leche y su producción diaria mostraron cambios significativos en el periodo analizado, salvo por un ligero aumento en el tiempo, en el contenido de PC y de $\mathrm{UGG} / \mathrm{ha}$ ( $\mathrm{r} 2=0,09$ y 0,06 respectivamente). Los indicadores productivos son consistentes con los valores nutricionales de esta especie forrajera, aunque resulta fundamental adelantar estudios más precisos acerca de la sincronización entre la energía y el nitrógeno suministrados al rumen, con el fin de mejorar procesos de eficiencia de la fermentación (Castro et al., 2018).

\section{- Dichantium aristatum}

El pasto Angleton (Dichantium aristatum) es un forraje ampliamente distribuido en el trópico bajo colombiano por su fácil adaptación, resistencia a condiciones agro climatológicas adversas y su contenido de materia seca, por lo que se utiliza constantemente para la elaboración de heno. Tradicionalmente ha sido utilizado para el levante y la ceba de ganado de carne, en zona caribe colombiana como Córdoba y norte de Antioquia. 


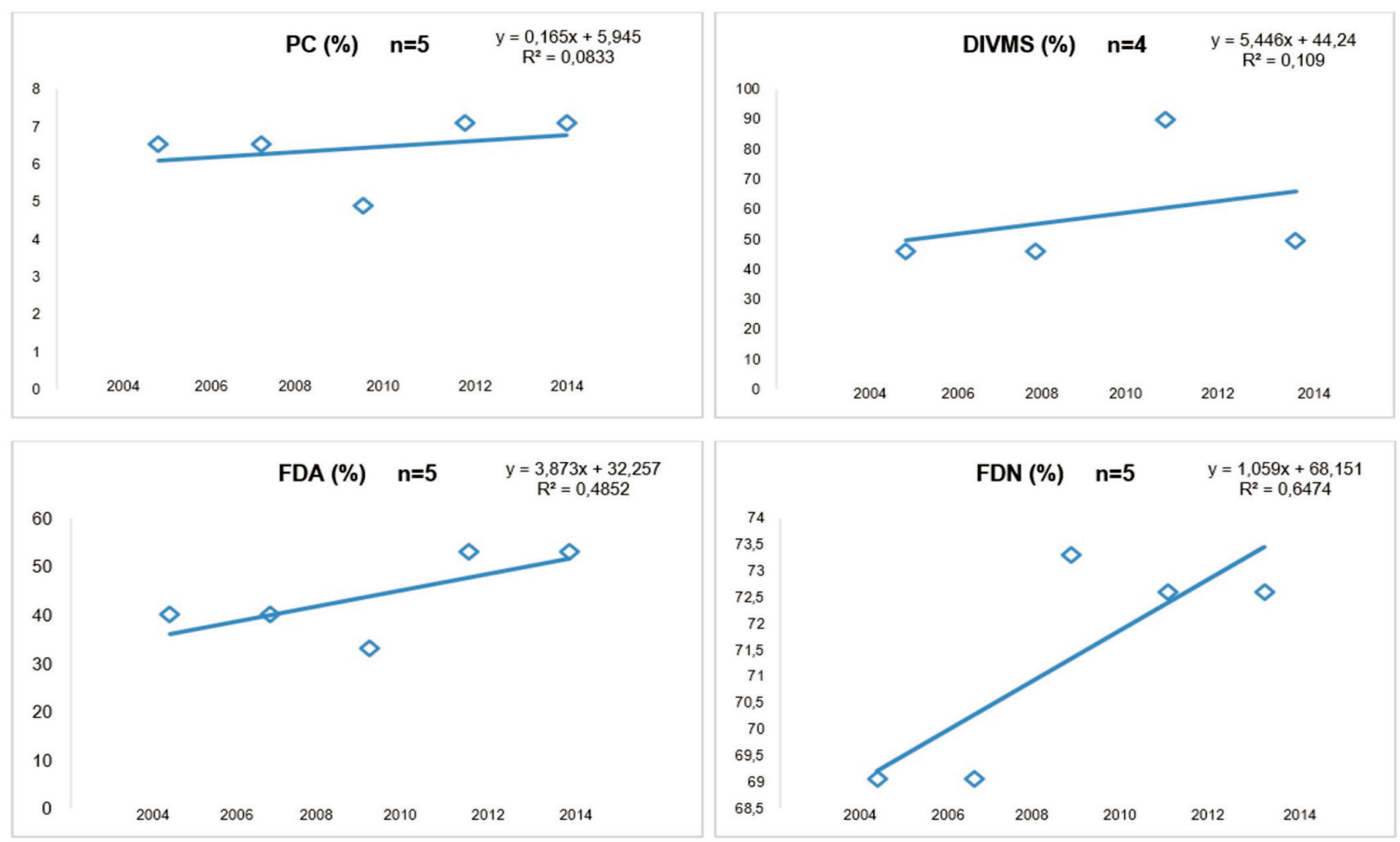

Figura 13. Variación en Fracciones Químicas de Dichantium Aristatum en Estudios publicados entre 1985 y 2015 en Colombia.

Fuente: el estudio 2018

Los reportes publicados para este forraje en los que se incluyera la composición y las variables productivas fueron pocos, aunque se obtuvieron datos productivos desde 1997 y composicionales desde 2004. Se observan algunas tendencias al incremento de FDN y FDA, que probablemente obedezcan al bajo número de observaciones, más que a una correlación que se pueda asociar al cambio en las fracciones químicas reportadas para el pasto Angleton. Debido a lo expuesto, los datos obtenidos para este forraje presentaron una alta variabilidad para el contenido medio de, DIVMS $(57,85 \pm 21,29)$ y $\operatorname{FDA}(43,87 \pm 8,79)$ y en menor proporción para FDN $(71,32 \pm 2,08)$ y PC $(6,44 \pm 0,91)$. Los datos promedios de PC encontrados en el estudio contrastan con los reportados por (Corpoica - Ahora Agrosavia - y Universidad Nacional de Colombia, 2013), con valores de 10 a $16 \%$. Por su parte, y según esta misma fuente, los reportes relacionados con DIVMS entre 65-75 \%, FDN $74 \%$ y FDA de $43,4 \%$, son similares a los datos reportados en los estudios en ventana de observación, con bajos indicadores de calidad. Este comportamiento deficiente, según García, et al., (2006), es responsable de la baja productividad de los rumiantes, sobre todo durante algunas épocas del año. 


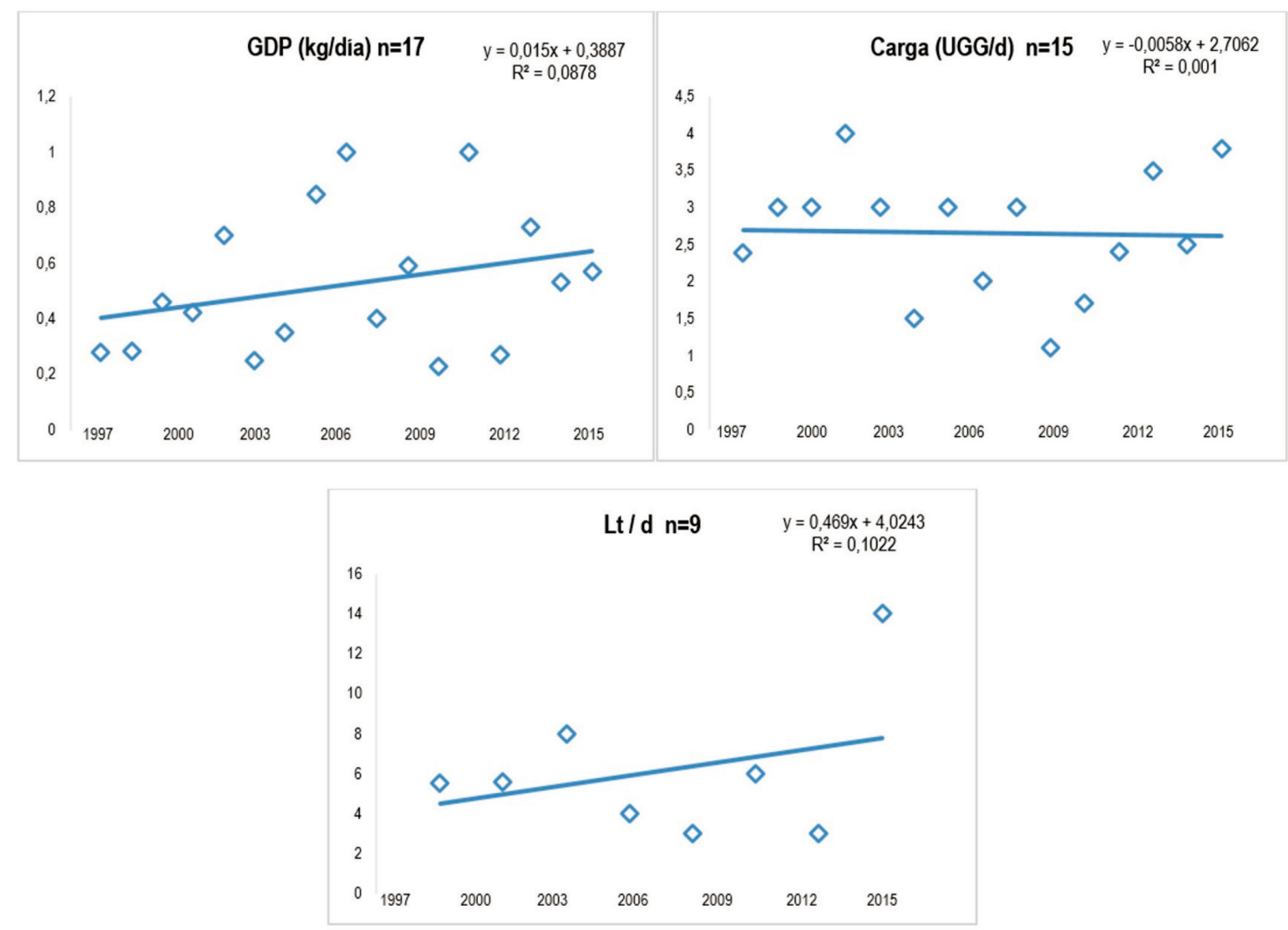

Figura 14. Reporte de la Ganancia diaria de peso, capacidad de carga (UGG/d) y Producción de leche (L/d) entre 1997 y 2015 en Colombia con Dichantium Aristatum.

Fuente: el estudio 2018

Las variables productivas reportadas en investigaciones que han estudiado el Angleton se asocian con sistemas de engorde bovino, en este sentido se obtuvo un mayor número de estudios en los que se reporta la GDP y la capacidad de carga, que la producción de leche o su composición. Aunque no fue estadísticamente significativa, se observó una ligera tendencia $(r 2=0,10)$ positiva para la producción de leche, no así para GDP $(r 2=0,08)$ ni para UGG/ha ( $r 2=0,001)$, esta ausencia en cambios detectables en el tiempo puede estar asociado a la restricción en el número de datos en estudios anteriores a 1997 lo cual no permite apreciar el comportamiento en el lapso de 30 años que se analizó con otros forrajes.
Se destaca la capacidad del forraje para ser utilizado en el engorde de bovinos y su potencial para sostener un alto número (comparado con otras gramíneas de pastoreo para trópico bajo) de animales por hectárea (en promedio 2,65 UGG/ha) con ganancias de peso históricas (en 12 años y 17 reportes incluidos en el estudio) que superan los $500 \mathrm{~g} / \mathrm{d}$. Estudios reportados por Torregroza, Cuadrado y Pérez (2006), corroboran este hallazgo, ya que registran datos de ganancias correspondientes a $660 \mathrm{~g} / \mathrm{d}$ en animales Romosinuano y $728 \mathrm{~g} / \mathrm{d}$ para animales Cebú.

\section{- Paspalum sp}

A pesar del bajo número de registros para la composición química especifica de este forraje 
y las variables productivas asociadas al uso exclusivo (no como parte de una mezcla de praderas), publicadas en los últimos 30 años, se incluyó esta especie forrajera, en el análisis, dada su importancia como grama nativa, presente en las principales sabanas de trópico bajo, en donde se levantan y engordan bovinos en el país.

Los reportes incluidos en este análisis comprenden desde 1987 a 2013, sin embargo, el género Paspalum, como base alimenticia de rumiantes de las sabanas nativas (piedemonte llanero y algunas zonas de la costa caribe), es subestimado como recurso forrajero por su bajo nivel de aporte nutricional (baja proteína, alta fibra y baja digestibilidad), e incluso es considerado a menudo como arvense en los procesos de introducción de gramíneas mejoradas (generalmente del género Brachiaria). En este sentido, muchos de los estudios lo reportan simplemente como pastura basal y en la gran mayoría de las investigaciónes no se realizan análisis de composición química. Su rusticidad, persistencia y adaptación a suelos de baja fertilidad se debe a un sistema radicular fuerte y profundo.

Así las cosas, a partir de 8 reportes entre 1988 y 2013 se obtuvo un contenido medio de $8,8 \%$ de PC, 66,24\% de FDN, 32,5 \% de FDA y $58,27 \%$ de DIVMS. Al respecto, Obrador (2007), expone que bajos niveles de proteína y de digestibilidad, como los reportados en sus estudios (< $8 \%$ y $50 \%$ respectivamente), interfieren en los niveles productivos que registran los animales.

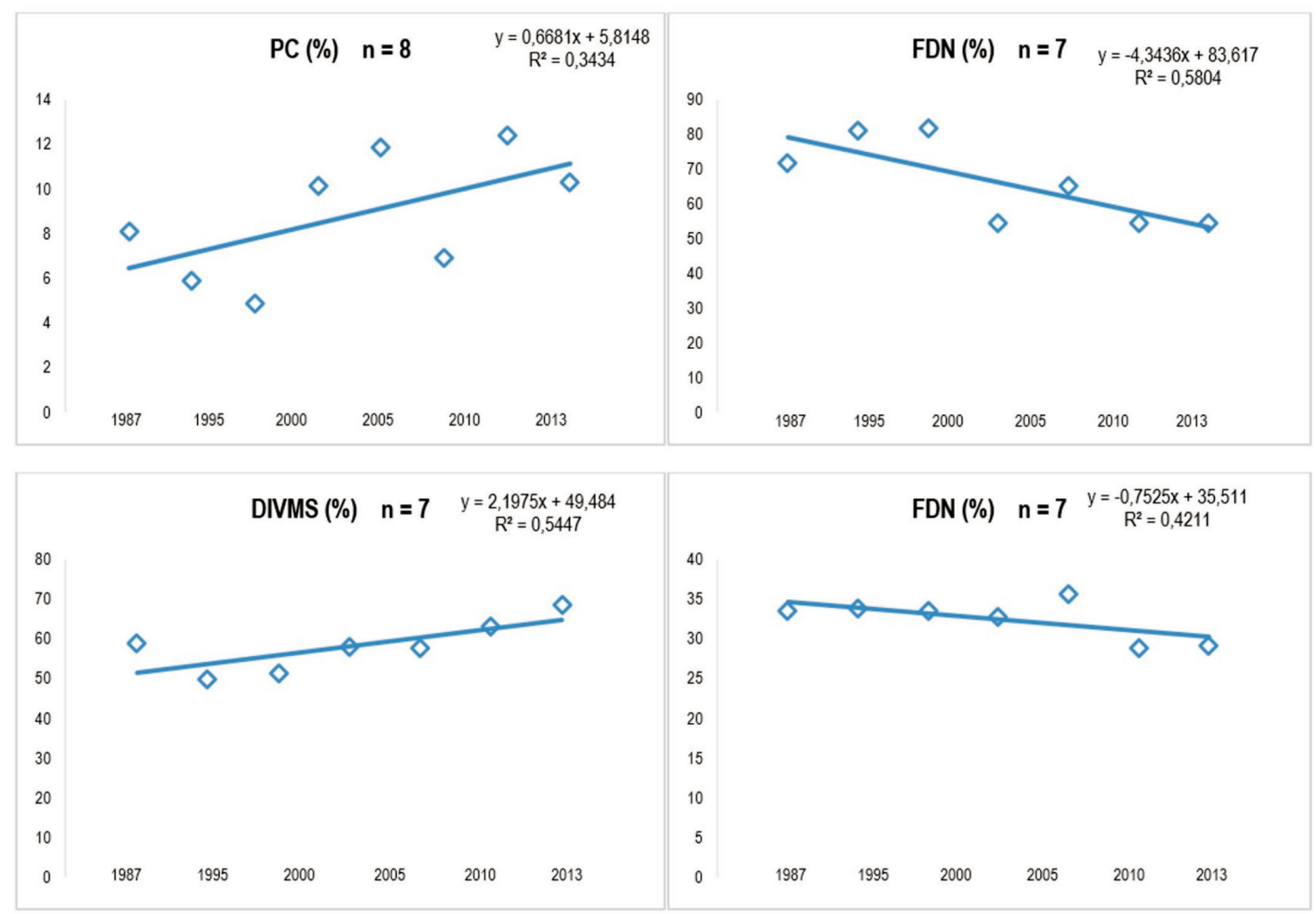

Figura 15. Variación en Fracciones Químicas de Paspalum Sp en Estudios publicados entre 1987 y 2013 en Colombia.

Fuente: el estudio 2018 
A pesar del bajo número de estudios para este forraje, se identifican tendencias generales de un aumento en la GDP $(r 2=0,58)$ y la capacidad de carga $(r 2=0,61)$ con una significancia estadística intermedia, indicando que en los últimos 26 años, los estudios reportan incrementos en el número de animales por hectárea e incrementos de peso en bovinos alimentados con grama nativa.

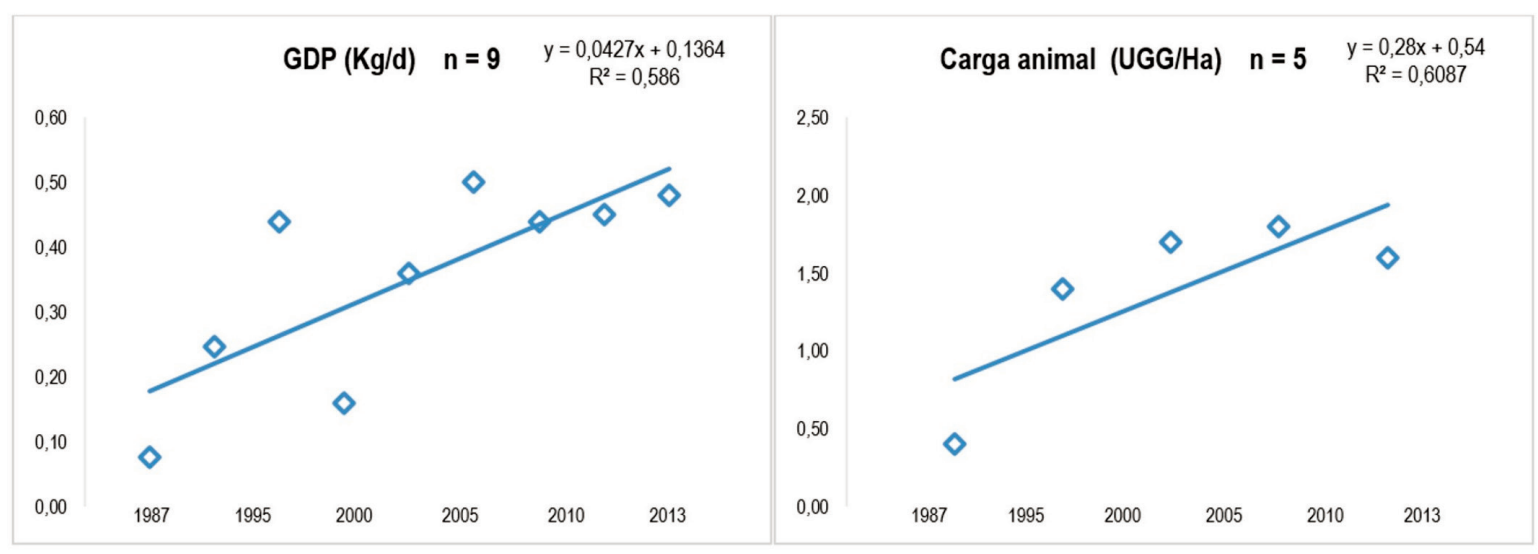

Figura 16. Reporte de la Ganancia diaria de peso, capacidad de carga (UGG/d) y Producción de leche (L/d) entre 1997 y 2015 en Colombia.

Fuente: el estudio 2018

\section{CONCLUSIONES}

El análisis comparado en el tiempo acerca del valor nutricional de los forrajes, y su respuesta en variables zootécnicas, constituye una herramienta muy importante para explicar y predecir el comportamiento nutricional de especies forrajeras y conocer el mejoramiento que se ha hecho en su calidad a través de los años.

En los estudios analizados, no se identificaron tendencias lineales ni un comportamiento lineal significativo a lo largo del tiempo, tanto para las variables asociadas a la calidad del forraje, como para las variables productivas, excepto las especies Brachiaria sp y Paspalum $s . p$, en las que se advierte una tendencia al aumento en la capacidad de carga entre los años estudiados UGG/ha ( $r 2=0,54$ y 0,53 respectivamente). A pesar de la baja significancia de la regresión se observó en las 9 especies un aumento sostenido en PC y DIVMS y reducción en FDA (salvo en Raygrass y Angleton).

Debido al comportamiento estacional de los forrajes en el trópico, no solo en la oferta sino en su calidad nutricional, es importante adelantar estudios históricos relacionados con la disponibilidad de biomasa, calidad e indicadores zootécnicos en diferentes regiones del país, incorporando al estudio, variables climáticas y edáficas, edad y parte de la planta, época del año y tecnologías aplicadas en manejo de praderas.

\section{LITERATURA CITADA}

Álvarez Perdomo, G., Vivas Moreira, R., Suárez Fernández, G., Cabezas Congo, R., Jacho Macías, T., Llerena Guevara, T., Valverde Moreira, H., Moreira Palacios, E., García Martínez, A., Chacón Marcheco, E., Verdecia Acosta, D. (2016). Componentes del rendimiento y composición química de Megathyrsus maximus en asociación con leguminosas. REDVET. Revista Electrónica 
de Veterinaria, 17 (12),1-12. Recuperado 12 de junio de 2017 de http://www.redalyc.org/ html/636/63649052025/

Anrique, R. (2014). Composición de alimentos para ganado bovino. Universidad Austral de Chile. Recuperado 13 de junio de 2018 de http://www. consorciolechero.cl/chile/documentos/composicion-de-alimentos-para-ganado-bovino.pdf

Barbosa, R. A., Nascimento Júnior, D. D., Vilela, H. H., Silva, S. C. D., Euclides, V. P. B., Sbrissia, A. F., y Sousa, B. M. D. L. (2011). Morphogenic and structural characteristics of guinea grass pastures submitted to three frequencies and two defoliation severities. Revista Brasileira de Zootecnia, 40 (5), 947-954. Recuperado 10 de mayo de 2018 de http://www.scielo.br/scielo.php?pid=S151635982011000500002\&script=sci_arttext

Beltrán, S., Hernández, A., García, E., Pérez, J., Kohashi, J., Herrera, J., Quero, A. y González, S (2005). Efecto de la altura y frecuencia de corte en el crecimiento y rendimiento del pasto Buffel (Cenchrus ciliaris) en un invernadero. Agrociencia, 39(2), 137-147. Recuperado 26 de abril de 2018 de https://www.colpos.mx/agrocien/Bimestral/2005/mar-abr/art-2.pdf

Brizuela, E., Ferrando, C., y Blanco, L. (2005). Distribución vertical de hojas y de la relación hoja-tallo en Trichloris crinita diferida. In Congreso Argentino de Producción Animal. 28. 200510 19-21, 19 al 21 de octubre de 2005. Bahía Blanca. AR.

Castro-Hernández, H., Domínguez-Vara, I. A., Morales-Almaráz, E., \& Huerta-Bravo, M. (2017). Composición química, contenido mineral y digestibilidad in vitro de Raigrás (Lolium perenne) según intervalo de corte y época de crecimiento. Revista Mexicana de Ciencias Pecuarias, 8 (2), 201-210. Recuperado 13 de junio de 2018 de http://cienciaspecuarias.inifap.gob.mx/index. php/Pecuarias/article/view/4445/3735

Carulla J E, Cárdenas E, Sánchez N y Riveros C. (2004). Valor nutricional de los forrajes más usados en los sistemas de producción lechera especializada de la zona andina colombiana; En: Eventos y Asesorías Agropecuarias EU (ed.), Seminario Nacional de Lechería Especializada: "Bases Nutricionales y su Impacto en la Productividad". Medellín, septiembre 1 y 2: 21 - 38.

Correa, H.J. (2011). Efecto del manejo del pastoreo y la suplementación alimenticia en vacas lactantes de sistemas especializados sobre su metabolismo energético y proteico y el contenido de proteína en la leche. Tesis de Doctorado en Ciencias de la Producción Animal, Universidad Nacional de Colombia, Sede Bogotá, Facultad de Medicina Veterinaria y de Zootecnia

Denda, S. S. (2017). Impacto de la fertilización nitrogenada sobre la producción y la composición química de trigo doble propósito y otros forrajes invernales: revisión bibliográfica. Ciencia Veterinaria, 7 (1), 65-81. Recuperado 9 de mayo de 2018 de https://cerac.unlpam.edu.ar/index.php/ veterinaria/article/view/1930

Detmann, E.; Paulino, M.F.; Zervoudakis, J.T.; Cecon, P.R.; Valadares Filho, S.C.; Gonçalves, L.C.; Cabral, L.S. e Melo, A.J.N. (2004). Níveis de proteína bruta em suplementos múltiplos para terminação de novilhos mestiços em pastejo durante a época seca: desempenho produtivo e características de carcaça. Rev Bras Zootecn, 33, 169-180. Recuperado 18 de junio de 2018 de http://www. scielo.br/pdf/\%0D/rbz/v33n1/a21v33n1.pdf

Corpoica y Universidad Nacional de Colombia. (2013). Dichanthium aristatum (Pasto Angleton).

Del Pozo, P. P. (2004). Bases ecofisiológicas para el manejo de los pastos tropicales. Anuario Nuevo. Universidad Agraria de La Habana. Sitio Argentino de Producción Animal. Recuperado 4 de mayo de 2018 de http://www.produccion-animal. com.ar/produccion_y_manejo_pasturas/pastoreo\%20sistemas/30-bases_ecofisiologicas_manejo_pasturas_tropicales.pdf

Gándara, L., Borrajo, C. Fernández, J., Pereira, M (2017). Efecto de la fertilización nitrogenada y la edad del rebrote sobre el valor nutritivo de Brachiaria brizantha cv "Marandú". Revista de la Facultad de Ciencias Agrarias Unicuyo, 49 (1), 69-77. Recuperado 3 de junio de 2018 de http:// www.scielo.org.ar/scielo.php?script=sci_arttext \&pid=S1853-86652017000100006

García, F. A. L., Miranda, J. A., y Borge, W. A. C. (2017). Producción y calidad de forraje con enmiendas orgánicas en pastura (Brachiaria Brizantha), en la Costa Caribe Sur de Nicaragua. Revista Universitaria del Caribe, 18(1), 83-90. Recuperado 15 de mayo de 2018 de http://www. revistasnicaragua.net.ni/index.php/caribe

García, D.; Noda, Y.; Medina, M.; Martín, G. y Soca, M. (2006). La morera: Una alternativa viable para los sistemas de alimentación animal en el trópico. Av. en Inv. Agropecuaria, 10(1), 55-72. Recuperado 8 de junio de 2018 de http://ww.ucol.mx/ revaia/portal/pdf/2006/enero/5.pdf 
Elizondo-Salazar, J. A. (2017). Producción de biomasa y calidad nutricional de tres forrajes cosechados a dos alturas. Agronomía Mesoamericana, 28(2), 329-340 Recuperado 14 de mayo de 2018 de http://www.redalyc.org/revista.oa?id=4 37\&perfil=publicacion\&tipo= coleccion

Heywood, V. H. (2008). Challenges of in situ conservation of crop wild relatives. Turk. J. Bot, 32, 421-432. Recuperado 10 de junio de 2018 de http://journals.tubitak.gov.tr/botany/issues/bot08-32-6/bot-32-6-1-0809-11.pdf

Jaramillo D., Jaramillo M., Restrepo L., Saglimbeni S. (2014). Ceba de ganado angus en trópico alto con pasto kikuyo (Pennisetum clandestinum Exchiov.). Recuperado 13 de febrero de 2019 de http://bdigital.ces.edu.co:8080/repositorio/bitstream/10946/3874/1/Ceba_Ganado_Angus.pdf

Juárez, L. F. I., Montero, L. M. y Núñez, H. G. (2011). Limitaciones y potencial de los forrajes para mejorar la nutrición de bovinos en regiones tropicales. En: Genómica y modelación en los nuevos escenarios de la ganadería bovina tropical. Segundo Simposio Internacional. Universidad $\mathrm{Na}$ cional de Colombia. 49-69 pp

León, G. M.; Martínez, S. S. J.; Pedraza O. R. M. y González, P. C. E. (2012). Indicadores de la composición química y digestibilidad in vitro de 14 forrajes tropicales. Rev. Prod. Animal, 24(1),15. Recuperado 14 de mayo de 2018 de http:// go.galegroup.com/ps/anonymous?id=GALE\%7C A466297473\&sid = googleScholar\&v $=2.1 \& \mathrm{it}=r \& l i$ nkaccess $=$ abs \&issn $=02586010 \& p=A O N E \& s w=w$

Mahecha, L., Escobar, J P Suárez, J.F., y Restrepo, LF (2007). Tithonia diversifolia (hemsl) Gray (Botón de oro) como suplemento forrajero de vacas F1 (Holstein por cebú). Livestock Research for Rural Development. 19 (2), 1-4. Recuperado 25 de abril de 2018 de http://www.Irrd.cipav.org.co/ Irrd19/2/mahe19016.htm

Milera, M. (2013). Contribución de los sistemas silvopastoriles en la producción y el medio ambiente. Avances en Investigación Agropecuaria, 7(3), 7-24. Recuperado 18 de abril de 2018 de http:// ww.ucol.mx/revaia/portal/pdf/2013/sept/1.pdf

Obrador-Olán, P., \& Hernández-Sánchez, D., \& Aranda-Ibáñez, E., \& Gómez-Vázquez, A., \& Camacho-Chiu, W., \& Cobos-Peralta, M. (2007). Evaluación de los forrajes de morera Morus alba y Tulipán Hibiscus rosa-sinensis a diferentes edades de corte como suplemento para corderos en pastoreo. Universidad y Ciencia, 23 (2),
115-125. Recuperado 16 de mayo de 2018 de http://www.redalyc.org/html/154/15423203/

Ojeda, F., Pino- Bárbara N., Lamela, L., Santana, H, y Montejo, I. (2010). Estudio de un ciclo de ceba con una dieta integral a base de forraje y hollejo de cítrico: Efecto de la calidad del forraje. Pastos y Forrajes, 33(1). Recuperado 18 de junio de 2018, de http://scielo.sld.cu/scielo.php?script=sci_ arttext\&pid=S0864-03942010000100007\&Ing=es \&tIng=es.

Peruchena, C. (2007). Suplementación de bovinos en sistemas pastoriles. Forrajes y alimentación Congreso Ganadero del Norte Argentino, Congreso CREA. Recuperado 14 de marzo de 2018 de http://www.produccion-animal.com.ar.

Paciullo, D.C., Tavares de Castro, C., De Miranda Gomide, C. A., Rogério Martins, M., Ávila Pires M. Diaz Mülle, M., Deise Ferreira, X. (2011). Performance of dairy heifers in a silvopastoral system. Livestock Science, 141, 166-172. Recuperado 2 de mayo de 2018 de https://www.sciencedirect. com/science/article/pii/S1871141311001971

Peñaherrera, A. (2015). Producción y calidad forrajera de pasto Soboya (Panicum maximum Jacq) a diferentes edades y alturas de corte. Tesis de pregrado. Universidad de las fuerzas armadas, Santo Domingo de los Tsáchilas.

Posada, S., Ceron, J.M., Arenas, J., Hamedt, J., y Álvarez, A. (2014). Evaluación del establecimiento de Raigrás (lolim sp.) en potreros de kikuyo (Pennisetum clandestinum) usando la metodología de cero labranzas. Revista CES Medicina Veterinaria y Zootecnia, 8 (1), 26-35. Recuperado 13 de junio de 2018 de http://revistas.ces.edu.co/index.php/ mvz/article/view/2831/2062

Rivera, J E., Cuartas, C A., Naranjo, J. F, Tafur, O., Hurtado, E. A., Arenas1, F A., Chará, J., y Murgueitio, E. (2015). Efecto de la oferta y el consumo de Tithonia diversifolia en un sistema silvopastoril intensivo (SSPi), en la calidad y productividad de leche bovina en el piedemonte Amazónico colombiano. Livestock Research for Rural Development, 27 (10), 1-9. Recuperado 25 de abril de 2018 de https://www.researchgate.net/publication/282606478_Efecto_de_la_oferta_y_el_consumo_de_Tithonia_diversifolia_en_un_sistema_ silvopastoril_intensivo_SSPi_en_la_calidad_y_ productividad_de_leche_bovina_en_el_piedemonte_Amazonico_colombiano_Effect_of_an_in

Santana Pérez, Á. A., Pérez López, A., y Figueredo Acosta, M. E. (2010). Efectos del estado de 
madurez en el valor nutritivo y momento óptimo de corte del forraje Napier (Pennisetum purpureum Schum.) en época lluviosa. Revista mexicana de ciencias pecuarias, 1(3) 277-286. Recuperado 5 de mayo de 2018 de http://cienciaspecuarias.inifap.gob. $\mathrm{mx} /$ index.php/Pecuarias/article/view/1504/1499

Soto, C., Valencia, A., Galvis, R.D., y Correa, H.J.(2005). Efecto de la edad de corte y del nivel de fertilización nitrogenada sobre el valor energético y proteico del pasto kikuyo (Pennisetum clandestinum). Revista Colombiana de Ciencias Pecuarias, 18(1) 17-26. Recuperado 15 de mayo de 2018 de http://www.scielo.org.co/scielo.php?script=sci_arttext\&pid $=$ S0120-06902005000100003

Tobía, C, y Villalobos, E. (2004). Producción y valor nutricional del forraje de soya en condiciones tropicales adversas. Agronomía Costarricense 28(1), 17-25. Recuperado 3 de mayo de 2018 de http://www.redalyc.org/pdf/436/43628102.pdf

Torregroza, L., Reza, S., Suárez, E., Espinosa, M., Cuadrado, H., Pastrana, I., Mejía, S., Jiménez, N., Abuabara, Y., (2015). Producción de carne en pasturas irrigadas y fertilizadas de Brachiaria híbrido cv. Mulato II en el valle del Sinú. Corpoica Cienc. Tecnol. Agropecu. 16 (1), 131-138. Recuperado 30 de abril de 2018 de http://www.scielo. org.co/pdf/ccta/v16n1/v16n1a12.pdf

Treydet, A., Heitkonig, I., Prins, $\mathrm{H}$ and Ludwig, F., (2007). Trees improve grass quality for herbivores in African Savannas. Perspect. Plant Ecol. Evol. Syst. 8, 197-205. Recuperado 2 de mayo de 2018 de https:// ac.els-cdn.com/S1433831907000121/1-s2.0S1433831907000121-main.pdf?_tid=ce75354f28b4-45d4-bdab-4b6979d8f72d\&acdnat $=15293$ 36819_959ff2f92ca3a63a4206401a3e858e73

Torregroza, L., Cuadrado, H., Pérez, J. (2006). Producción de carne en novillos f1 Romo-Cebú con pasto Angleton (Dichanthium aristatum) ensilajes y suplementos en el valle del Sinú. Rev. MVZ Córdoba, 11 (2), 825-829. Recuperado 13 de junio de 2018 de http://www.scielo.org.co/pdf/ $\mathrm{mvz} / \mathrm{v} 11 \mathrm{n} 2 / \mathrm{v} 11 \mathrm{n} 2 \mathrm{a} 07 . \mathrm{pdf}$

Uvidia, H., Ramírez, J., Vargas, J., Leonard, I., \& Sucoshañay, J. (2015). Relación del clima con el rendimiento y la calidad del Pennisetum purpureum en la Amazonia Ecuatoriana. REDVET. Revista Electrónica de Veterinaria, 16(6), 1-10. Recuperado 25 de mayo de 2018 de http://www. redalyc.org/pdf/636/63641399005.pdf

Vasquez O., Smith TR. (2000). Factors Affecting Pasture Intake and Total Dry Matter Intake in Grazing Dairy Cows. Recuperado 13 de febrero de 2019 en https://www.journalofdairyscience. org/article/S0022-0302(00)75117-4/abstract

Verdecia DM., Herrera R S., Ramírez J L., Leonard I., Uvidia H., Álvarez Y., Paumier Z., Arceo Y., Santana, A. y Almanza, D. (2015). Potencialidades agroproductivas de dos cultivares de Megathyrsus maximus en la región oriental de Cuba. Revista electrónica de Veterinaria, 16 (11).1-9. Recuperado 30 de marzo de 2018 de http://www.veterinaria.org/revistas/redvet/n111115/111501.pdf

Villalobos, L y Sánchez, J.M. (2010). Evaluación agronómica y nutricional del pasto Rygrass perenne tetraploide producido en las lecherías de las zonas altas de Costa Rica, producción de biomasa y fenología. Agronomía Costarricense, 34(1), 31-42. Recuperado 13 de junio de 2018 de http://www. redalyc.org/pdf/436/43617800004.pdf

Veloza, J. (2008). Evaluación económica y nutricional de un programa complementario de la fertilización edáfica a base de aminoácidos y calcio de aplicación foliar en praderas de kikuyo - Ryegras en la sabana de Bogotá. Trabajo de grado para optar al título de Zootecnista. Universidad de la Salle. Bogotá.
Conflicto de Intereses Los autores declaran no tener ningún conflicto de intereses

\section{(C) (1) $\Theta(0$}


